\title{
High performance thin-film nanofibrous composite hemodialysis membranes with efficient middle-molecule uremic toxin removal
}

\author{
Xufeng Yu ${ }^{\mathrm{a}}$, Lingdi Shen ${ }^{\mathrm{a}}$, Yadong Zhu ${ }^{\mathrm{a}}$, Xiong Li ${ }^{\mathrm{a}}$, Yin Yang ${ }^{\mathrm{a}}$, Xuefen Wang ${ }^{\mathrm{a} *}$, \\ Meifang Zhu ${ }^{\mathrm{a}}$, Benjamin S. Hsiao ${ }^{\mathrm{b}}$ \\ ${ }^{a}$ State Key Lab for Modification of Chemical Fibers and Polymer Materials, Donghua \\ University, Shanghai, 201620, P.R. China \\ ${ }^{b}$ Department of Chemistry, Stony Brook University, Stony Brook, N Y 11794, USA
}

*Corresponding author. Tel.: 86-21-67792860; Fax: 86-21-67792855.

E-mail address: wangxf@dhu.edu.cn 


\begin{abstract}
Membrane structure design is critical for the development of high-performance hemodialysis membranes. Here, a thin-film nanofibrous composite (TFNC) membrane, consisting of a two-tier composite structure, i.e., an ultrathin hydrophilic separation layer of chemically cross-linked polyvinyl alcohol (PVA), and an electrospun polyacrylonitrile (PAN) nanofibrous supporting layer, was demonstrated as the hemodialysis membrane for the first time. The optimized PVA/PAN TFNC membrane exhibited high permeability $\left(\sim 290.5 \mathrm{~L} / \mathrm{m}^{2} \mathrm{~h}\right.$ at $\left.0.1 \mathrm{MPa}\right)$ and excellent selectivity which should be attributed to its unique structure with ultrathin separation layer and highly porous supporting layer. In addition, the TFNC membrane also possessed excellent overall mechanical properties, good hydrophilicity and comparable hemocompatibility properties (protein adsorption, platelet adhesion, complement activation, hemolysis ratio). The hemodialysis simulation experiments on optimized TFNC membrane showed that $82.6 \%$ of urea and $45.8 \%$ of lysozyme were cleaned and $98.8 \%$ of bovine serum albumin (BSA) was retained. The TFNC membranes exhibited excellent hemodialysis performances, especially for the middle-molecule uremic toxin removal, which was more efficient than conventional hemodialysis membranes reported so far, suggesting PVA/PAN TFNC membranes as promising alternatives for hemodialysis applications.
\end{abstract}

Keywords: Thin-film nanofibrous composite membrane; Hemodialysis; Two-tier structure; Selectivity. 


\section{Introduction}

Chronic/acute kidney disease is a significant cause of mortality in the world, and the disease's incidence rate has increased rapidly because of the aging population and the rising number of diabetes and hypertension patients. Globally, the number of kidney disease patients was estimated to be 600 million and the number of patients worldwide is now growing by $6-7 \%$ annually [1]. When the concentration of uremic toxic increases beyond the standard range in blood, unwanted side effects will occur and this is named as uremic syndrome. The great majority of patients require dialysis to prolong life and the market of hemodialysis membranes currently worth more than US\$1 billion annually [2]. Hemodialysis is a vital clinical therapy for the patients with renal diseases. It is the process of removing blood from a patient, purifying that blood through the hemodialysis equipment (also called as artificial kidney), and then returning it to the patient's blood stream [3]. The core element of a hemodialysis equipment is the hemodialysis membrane, which can eliminate toxic metabolites and excess water from blood via diffusive and convective transport across the membrane and prevent loss of necessary proteins due to pore size exclusion of the membrane [4]. Therefore, the development of high-performance hemodialysis membranes has become a pivotal issue in hemodialysis treatment to enhance the quality of treatment and increase the patients' longevity.

Uremic toxins can be categorized into two groups [5]: small-molecular weight uremic toxin $(<2,000 \mathrm{Da})$, such as urea and creatinine; middle-molecule uremic toxin (ranging in molecular weight from 2,000 to 60,000 Da), such as parathyroid hormone $(9.5 \mathrm{kDa}), \beta_{2}$-microglobulin $(11.8 \mathrm{kDa})$ and pro-inflammatory cytokines IL-1 $\beta$ (17 $\mathrm{kDa})$. Although the hemodialysis has certainly changed the prognosis of chronic/acute kidney disease, the mortality rates of the patients with renal diseases remain unacceptably high (exceeding 20\%) [6]. The substantial shortcoming, which the hemodialysis membranes are inadequate to remove middle-molecules, results in the disappointing outcomes for hemodialysis patients [7].

Mass transfer through the hemodialysis membrane is essentially controlled by the 
material and structure of the membrane. At present, most relevant hemodialysis membranes are prepared by classical non-solvent induced phase separation (NIPS) process. Hemodialysis membranes prepared according to these methods suffer from a typical tradeoff: it is paradoxical to reconcile the desire of high removal of middle-molecular toxic substances and high retention of big proteins simultaneously because of their broad pore size distribution. As a result, commercial and recently developed hemodialysis membranes are suffering severely from the problem that it is inadequate to remove middle-molecule uremic toxin while retaining big albumin. Cellulose membranes are widely used for dialysis because of their effective removal for small uremic toxins. However, these membranes could not reduce or even increase the concentration of middle-molecule toxin due to their homogeneous and dense structure after hemodialysis process [8-11]. In attempt to remove middle-molecules efficiently, synthetic polymers membranes, such as polyacrylonitrile (PAN), polysulfone (PSf), polyethersulfone (PES), polyvinylidene difluoride (PVDF), and polylactic acid (PLA), with larger pore sizes and high flux compared to cellulosic membranes were developed [12-17]. However, these membranes still provided low clearance for middle-molecule toxin (1.2\% of middle-molecules could be cleaned out for pure PES membrane after simulating dialysis for $4 \mathrm{~h}$ and $12.6 \%$ for pure PLA membrane) $[18,19]$. In order to maximize the removal of middle molecules toxin and achieve the desired outcome as efficient as the natural kidney, the main focus of recent membrane development has been to further increase pore size and improve the permeability via surface grafting, coating or blending [20-24]. The integration of the nanocomposite dialysis membranes were the recent modifications done to improve porosity, water flux and efficiency $[25,26]$. Inevitably, the broad pore size distribution at large mean pore sizes would cause undesirable big albumin permeation [27]. To date, the toxic clearance ability for middle molecular toxic substances of these membranes was still not high $(\sim 30 \%)$, and the loss of albumin was increased concurrently (albumin retention decrease to $\sim 90 \%$ ) [28]. It is difficult to accomplish the desire due to the balance between the elimination of small/middle molecular toxic substances and the retention of large good proteins. Therefore, there is a great need 
for innovative hemodialysis membranes with better characteristics such as high removal of middle molecular toxic substances and high retention of big proteins simultaneously.

Hemodialysis process is essentially an interfacial issue, and thus the key point of hemodialysis is the membrane structural design for high-performance. As we know, highly porous nanofibrous membranes possess high permeability and completely interconnected pores that make them appropriate for a wide range of filtration applications [29]. Actually, it has been demonstrated that highly permeable nanofibrous membranes are excellent candidates as the microfiltration membranes or as the microporous supporting substrates under the top barrier layer to form high flux thin-film nanofibrous composite membranes (TFNC) [30-33]. In our previous studies, TFNC membranes containing a barrier layer supported by a nanofibrous substrate have been demonstrated for ultrafiltration, the as-prepared TFNC membranes could perform high flux $\left(347.8 \mathrm{~L} / \mathrm{m}^{2} \mathrm{~h}\right)$ with high organic rejection (> 99.5\%) at $0.2 \mathrm{MPa}$ feeding pressure in filtrating oil emulsions with sizes between 10-50 $\mathrm{nm}$ [34] or high performance in bovine serum albumin (BSA) filtration with water flux $173.0 \mathrm{~L} / \mathrm{m}^{2} \mathrm{~h}$ and rejection above $98.0 \%$ at feeding pressure $0.3 \mathrm{MPa}$ [35]. As a result, the above kinds of TFNC membranes after optimized preparation could be good candidates for hemodialysis with high protein retention and small/middle molecular toxic substances leakage. Polyvinyl alcohol (PVA) with good hydrophilicity and biocompatibility, is often used as a basic material for a variety of biomedical applications, like dressing skin and artificial organs $[36,37]$. Therefore, PVA is an excellent material for the active separation layer of the hemodialysis membrane. Herein, the PVA/PAN TFNC membrane has been attempted as the hemodialysis membrane for the first time. The schematic of the fabrication process was shown in Scheme 1. The morphology, permeability, selectivity, hydrophilicity, mechanical property, protein adsorption, platelet adhesion, complement activation, hemolysis, and hemodialysis of the TFNC membrane were investigated and compared with the benchmark flat membrane in details. The objective of this work was to develop a new kind of PVA/PAN TFNC membranes with high permeability and excellent selectivity for potential application 
in hemodialysis.

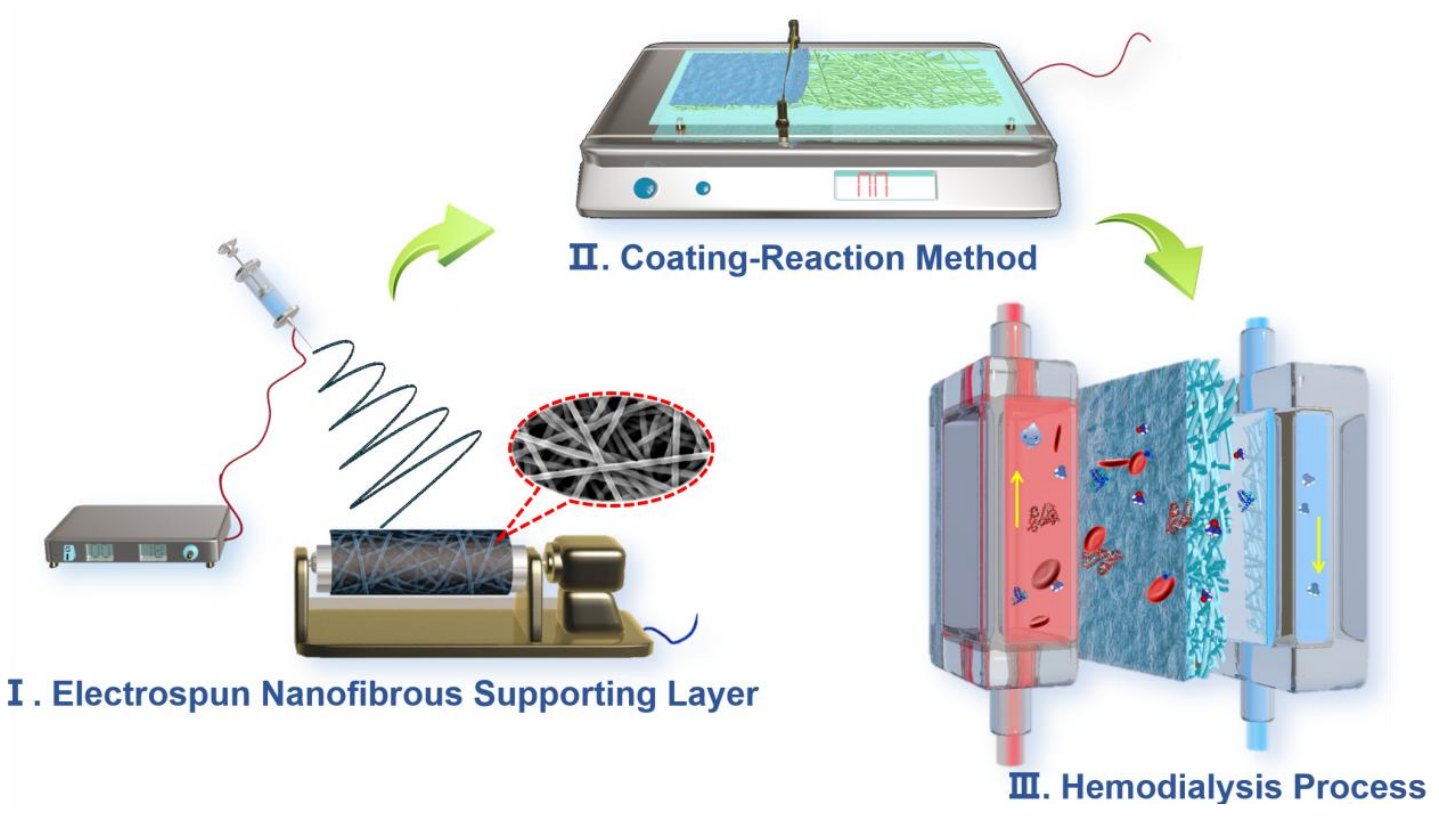

Scheme 1 Illustration for the fabrication procedure of two-tier TFNC membranes based on PAN electrospun nanofibrous scaffold support and PVA hydrogel coating for hemodialysis.

\section{Experimental Details}

\subsection{Materials}

PAN with a weight average molecular weight $\left(\mathrm{M}_{\mathrm{w}}\right)$ of $120,000 \mathrm{~g} / \mathrm{mol}$ was supplied by Shanghai Jinshan Chemical Co., Ltd. PVA $\left(\mathrm{M}_{\mathrm{w}}=146,000-186,000 \mathrm{~g} / \mathrm{mol}\right.$, degree of hydrolysis $=88 \%$, Sigma Aldrich) was used as the active separation layer material. PSf (P-1700) was used as the control material and was obtained from Solvay Shanghai Co., Ltd. N, N'-dimethylformamide (DMF), glutaraldehyde (GA), hydrochloric acid $(\mathrm{HCl})$, boric acid, and p-dimethylaminobenzaldehyde (PDAB) were bought from Sinopharm Chemical Reagent Co., Ltd., China. Urea, creatinine, vitamin $\mathrm{B}_{12}$, lysozyme, and bovine serum albumin (BSA, fraction $\mathrm{V}$ ) were purchased from J\&K, China. Micro BCA ${ }^{\mathrm{TM}}$ protein assay reagent kit was the product of PIERCE. C3a, C5a ELISA kits and anticoagulant blood were obtained from Shanran Biotechnology 
Co., Ltd, China. All chemicals were used as received without further purification.

For comparison tests, the benchmark PSf membrane was selected for comparison with our TFNC membrane. Synthetic polymers are used by the clinic in most of the hemodialysis membranes, 71\% of which are derived from PSf [38]. Commercial PSf hemodialysis membranes are always in hollow fiber configuration and they were widely acknowledged as providing an optimal properties in terms of solute removal and hemocompatibility. But for the lab testing, flat sheet membrane was necessary. Therefore benchmark PSf flat sheet (home-made) membrane was prepared based on a conventional membrane prepared via phase inversion method $[12,14,39,40]$.

\subsection{Fabrication of PVA/PAN TFNC membranes for hemodialysis}

\subsubsection{Preparation of PAN nanofibrous supporting layer}

PAN nanofibrous scaffold was prepared by electrospinning technique according to our previous report [34]. It was prepared using a laboratory setup electrospinning equipment. In brief, a homogeneous solution (8 wt\%) of PAN in DMF was prepared at $50{ }^{\circ} \mathrm{C}$ for $24 \mathrm{~h}$. The applied electric voltage was $24 \mathrm{kV}$ and the solution feed rate was $16 \mu \mathrm{L} / \mathrm{min}$. The relative humidity and temperature were kept between $45-50 \%$ and $25-30{ }^{\circ} \mathrm{C}$ respectively. To improve the mechanical strength and provide smooth surface for the preparation of top separation layer, the PAN nanofibrous scaffold used as the supporting layer was cold-pressed at room temperature at $5 \mathrm{MPa}$ for $60 \mathrm{~s}$.

\subsubsection{Preparation of the PVA/PAN TFNC membranes by coating-reaction method for} hemodialysis

Inspired by the coating-reaction method in the literature [41], the TFNC membranes were optimized for hemodialysis application. An aqueous PVA solution (1-2.5 wt\%) was prepared by dissolving PVA in ultrapure water under stirring at $70{ }^{\circ} \mathrm{C}$ for $6 \mathrm{~h}$. An $\mathrm{HCl}(1.5 \mathrm{M})$ solution was added to adjust the $\mathrm{pH}$ level $(\sim 2)$, then a GA solution was subsequently added to initiate the situ cross-linking reaction and the gelling time was monitored before coating process. The crosslinking degree of the 
PVA coating layer was varied by changing the molar ratio of GA and PVA repeat unit. For preparation of two-tier TFNC membranes, the PAN supporting layer was soaked in a $0.8 \mathrm{M}$ boric acid solution in order to enhance the adhesion between the supporting and separation layer by crosslinking between boric acid and hydroxyl groups of PVA [34]. The PVA/GA solution was cast onto the wet PAN supporting layer using an automated applicator after a suitable reaction time to prevent the solution penetration. The resulting two-tier TFNC membrane was incubated for $6 \mathrm{~h}$ at room temperature to complete the cross-linking reaction and then washed with ultrapure water for three days to eliminate the residual boric acid and GA molecules thoroughly, and then stored in a water bath before use.

\subsection{Characterization of PVA/PAN TFNC hemodialysis membranes}

\subsubsection{Membrane morphologies}

The morphology of membranes was examined via scanning electron microscopy (SEM, JSM-5600LV, Japan). The diameter of nanofibers was measured using ImageJ analysis software. The cross-sectioned membranes were prepared by fracturing in liquid nitrogen and all samples were gold coated before imaging.

\subsubsection{Membrane transport properties}

The pure water permeation (PWP) flux and protein rejection experiments were measured by a cross flow cell with an effective membrane area of $30 \mathrm{~cm}^{2}$. The PWP was recorded at $0.1 \mathrm{MPa}$ until a stable water flux value obtained and was determined using the equation (1):

$$
\mathrm{PWP}=\frac{\mathrm{Q}}{\Delta \mathrm{t} \times \mathrm{A}}
$$

where PWP represented the flux $\left(\mathrm{L} / \mathrm{m}^{2} \mathrm{~h}\right)$ for pure water, $\mathrm{Q}$ was the volume of permeation solution (L) and $\Delta \mathrm{t}$ was the permeation time (h). Moreover, BSA solution was used as feed solution with the concentration of $1 \mathrm{mg} / \mathrm{mL}$. The BSA concentration was estimated with a UV-vis spectrophotometer (Purkinje, model TU-1950, Beijing, 
China) at a wavelength of $280 \mathrm{~nm}$. Whereas the rejection rate was calculated by:

$$
\mathrm{R}=\frac{\mathrm{C}_{\mathrm{f}}-\mathrm{C}_{\mathrm{p}}}{\mathrm{C}_{\mathrm{f}}} \times 100 \%
$$

where $C_{p}$ and $C_{f}$ were the concentrations of the permeation and feed solution respectively.

The membrane sieving coefficient (SC) was also determined using the cross flow cell. The following compounds were dissolved in ultrapure water with initial concentrations of $1.5 \mathrm{mg} / \mathrm{mL}$ urea, $0.1 \mathrm{mg} / \mathrm{mL}$ creatinine, $0.1 \mathrm{mg} / \mathrm{mL}$ vitamin $\mathrm{B}_{12}, 0.4$ $\mathrm{mg} / \mathrm{mL}$ lysozyme, $1 \mathrm{mg} / \mathrm{mL}$ BSA and pressurized through the membrane at $0.1 \mathrm{MPa}$. Then the concentrations of the feed and permeation were analyzed using the UV-vis spectrophotometer. The SC was calculated with the following equation:

$$
\mathrm{SC}=\frac{\mathrm{C}_{\mathrm{p}}}{\mathrm{C}_{\mathrm{f}}}
$$

where $C_{p}$ and $C_{f}$ were the concentrations of the permeation and feed solution respectively. $\mathrm{SC}=1$ meant that the substance passed freely through the membrane, while $\mathrm{SC}=0$ meant that the membrane rejected the substance completely. Three samples were tested for each type of membrane so as to ensure the reproducibility of the data.

\subsubsection{Hydrophilicity measurements}

The hydrophilicity of the membranes was performed by a dynamic contact angle testing instrument (OCA40, Dataphysics, Filderstadt, Germany). The membrane was mounted on the sample holder and $2.0 \mu \mathrm{L}$ distilled water was dropped onto the air-side surface of the membrane at room temperature. Average water contact angles were obtained by measuring the sample at five different positions.

\subsubsection{Mechanical property}

The tensile stress and tensile strain of the resultant membranes were measured on a tensile testing machine (WDW3020, Changchun, China). For the tensile test, the gauge length was $50 \mathrm{~mm}$ and the width was $15 \mathrm{~mm}$. The chosen cross-head speed was 
$10 \mathrm{~mm} / \mathrm{min}$. All measurements were carried out at room temperature and the set of experiments consisted of five repetitions for each sample.

\subsubsection{Blood compatibility}

\subsubsection{Protein adsorption}

Protein adsorption to the membranes was measured following the technique of Deng et al [42]. Firstly, the membrane was placed in 12 -well plates with $1 \mathrm{mg} / \mathrm{mL}$ BSA in phosphate buffered saline (PBS, $\mathrm{pH}=7.4$ ) solution and incubated at $37^{\circ} \mathrm{C}$ for $1 \mathrm{~h}$. After subsequently rinsing with ultrapure water slightly, the membrane was placed in solution $2 \mathrm{wt} \%$ sodium dodecyl sulfate (SDS) solution at $37{ }^{\circ} \mathrm{C}$ and shaken for $1 \mathrm{~h}$ to remove the adsorbed protein. The protein concentration was determined by Micro $\mathrm{BCA}^{\mathrm{TM}}$ protein assay reagent kit. The adsorbed protein amount on the membrane surface was calculated using the equation (4):

$$
\text { Adsorptionamount }\left(\mu \mathrm{g} / \mathrm{cm}^{2}\right)=\frac{\mathrm{C} \times \mathrm{V}}{\mathrm{S}}
$$

where $\mathrm{C}$ was the protein concentration in the SDS solution, $\mathrm{V}$ was the volume, and $\mathrm{S}$ was the area of membrane. The given value was averaged from five different measurements, and the data were expressed as mean \pm standard deviation (SD).

\subsubsection{Platelet adhesion}

Platelet-rich plasma (PRP) was obtained by anticoagulant blood at $1500 \mathrm{rpm}$ for 20 min and used to study the platelet adhesion on the surfaces of the membranes [19]. The membrane $(1 \mathrm{~cm} \times 1 \mathrm{~cm})$ was immersed with PRP in each well of the culture plate and equilibrated at $37{ }^{\circ} \mathrm{C}$ for $1 \mathrm{~h}$. After that, the PRP was removed and the membrane was rinsed at least three times with PBS solution. The adhering platelets were fixed with $2.5 \mathrm{wt} \% \mathrm{GA}$ in PBS for 1 day at $4{ }^{\circ} \mathrm{C}$. Finally, the membrane was washed with PBS solution and passed through a series of graded alcohol-PBS solutions $(25 \%, 50 \%, 75 \%, 85 \%, 90 \%, 95 \%$, and $100 \%)$ to be dried. SEM images were recorded using a scanning electron microscope (JSM-5600LV, Japan) to 
investigate the platelet adhesion on the membranes.

\subsubsection{Complement activation}

Commercial enzyme-linked immunosorbent assays (ELISA) were used to investigate the complement activation [43]. The whole anticoagulant blood incubated with the membrane for $1 \mathrm{~h}$ and centrifuged for 20 minutes at $4{ }^{\circ} \mathrm{C}$ to obtain plasma. Then the plasma was mixed with specific inhibitors according to the respective instruction manuals for each assay. The experiments were repeated to ensure reliability. The data were expressed as mean \pm SD and analyzed by statistical methods (significant difference, $\mathrm{p}<0.05$ ).

\subsubsection{Hemolysis assay}

Hemolysis ratio (HR) was carried out to evaluate the red blood cell compatibility of the membranes [44]. Samples were rinsed with ultrapure water, and then soaked in normal saline at $37{ }^{\circ} \mathrm{C}$ for $30 \mathrm{~min}$. Whole blood was added to the normal saline, maintaining $37^{\circ} \mathrm{C}$ for $1 \mathrm{~h}$. After centrifuging at $1500 \mathrm{rpm}$ for $10 \mathrm{~min}$, light absorbance was measured at $540 \mathrm{~nm}$ on the supernatant to analyze the free hemoglobin concentration. Ultrapure water and normal saline were used as positive and negative controls. The HR was calculated using the following equation:

$$
\mathrm{HR}(\%)=\frac{\mathrm{AS}-\mathrm{AN}}{\mathrm{AP}-\mathrm{AN}} \times 100 \%
$$

where AS was the absorption value of samples, AN and AP were the absorption value of negative and positive control respectively. The average was used and the error was no more than $0.2 \%$.

\subsubsection{Dialysis simulation experiment in vitro}

The schematic diagram in Fig. 1 showed the lab-scale single layer dialysis test system utilized to evaluate the dialysis performance of membranes as described by Idris et al [10]. This dialyzer set-up maximized the concentration difference across the 
membrane and ensured maximum solute transfer. The dialysis test cell had an effective area of $30 \mathrm{~cm}^{2}$ and was divided between two separate compartments. The solution reservoir was filled with a mixture of solutes containing urea $(1.5 \mathrm{mg} / \mathrm{mL})$, lysozyme $(0.04 \mathrm{mg} / \mathrm{mL})$ and BSA $(1 \mathrm{mg} / \mathrm{mL})$ in order to mimic the real condition of dialysis treatment. Urea was the major small molecular uremic toxic and lysozyme $(14 \mathrm{kDa})$ was used in place of middle molecular $\beta_{2}$-microglobin. The flow rates of solution reservoir and pure water reservoir were kept at $200 \mathrm{~mL} / \mathrm{min}$ and $500 \mathrm{~mL} / \mathrm{min}$ respectively. The temperature was maintained at $37{ }^{\circ} \mathrm{C}$ and $10 \mathrm{~mL}$ of samples were collected from solution reservoir for every $1 \mathrm{~h}$ interval for duration of $4 \mathrm{~h}$, and the experiments were repeated thrice. The change of urea concentration was monitored spectrophotometrically with the use of the color reaction between urea and PDAB, and that of BSA and lysozyme was measured with ultraviolet spectrophotometer at $278 \mathrm{~nm}$ and $280 \mathrm{~nm}$ respectively. The urea and lysozyme clearance was calculated using the equation (6):

Clearance percentage $(\%)=\frac{\mathrm{C}_{0}-\mathrm{C}_{\mathrm{t}}}{\mathrm{C}_{0}} \times 100 \%$

where $\mathrm{C}_{0}$ and $\mathrm{C}_{\mathrm{t}}$ were the urea or lysozyme concentrations in the solution reservoir at time $\mathrm{t}=0$ and $\mathrm{t}=1,2,3,4 \mathrm{~h}$, respectively. The BSA retention was calculated using the equation (7):

BSA retention percentage $(\%)=\frac{\mathrm{C}_{\mathrm{t}}}{\mathrm{C}_{0}} \times 100 \%$

where $\mathrm{C}_{0}$ and $\mathrm{C}_{\mathrm{t}}$ were the BSA concentrations in the solution reservoir at time $\mathrm{t}=0$ and $\mathrm{t}=1,2,3,4 \mathrm{~h}$, respectively.

The overall mass transfer coefficient $\mathrm{K}_{0}$ was determined directly from the solute mass balance between the two compartments [26]:

$\ln \left[\frac{\mathrm{C}_{1(\mathrm{t} 1)}-\mathrm{C}_{2(1)}}{\mathrm{C}_{1(\mathrm{t} 0)}-\mathrm{C}_{2(\mathrm{t})}}\right]=\mathrm{K}_{0} \mathrm{~S}\left[\frac{1}{\mathrm{~V}_{1}}-\frac{1}{\mathrm{~V}_{2}}\right]\left(\mathrm{t}_{0}-\mathrm{t}_{1}\right)$

where $\mathrm{C}_{1}$ and $\mathrm{C}_{2}$ were the concentrations at sampling times, $\mathrm{S}$ was the effective membrane area $\left(30 \mathrm{~cm}^{2}\right)$, and $V_{1}$ and $V_{2}$ were the solution volumes in each reservoir.

The overall resistance to mass transfer is composed of three components, the blood compartment resistance, the membrane resistance and the dialysate compartment 
resistance. It is expressed according to a series resistance model [45]:

$\frac{1}{\mathrm{~K}_{0}}=\frac{1}{\mathrm{~K}_{\mathrm{B}}}+\frac{1}{\mathrm{P}_{\mathrm{M}}}+\frac{1}{\mathrm{~K}_{\mathrm{D}}}$

Due to Raff [46], the blood side and dialysate side mass transfer coefficients $K_{B}$ and $\mathrm{K}_{\mathrm{D}}$ depend on module geometry, fluid properties and operating conditions:

$$
\begin{aligned}
& \mathrm{K}_{\mathrm{B}}=1.62\left(\operatorname{Re}_{\mathrm{B}} \times \mathrm{Sc}_{\mathrm{B}}\right)^{1 / 3} \times \mathrm{D}_{\mathrm{B}} \\
& \mathrm{K}_{\mathrm{D}}=\mathrm{a}\left(\operatorname{Re}_{\mathrm{D}}\right)^{\mathrm{b}} \times \mathrm{D}_{\mathrm{D}}
\end{aligned}
$$

Here, Re is the Reynolds number, Sc is the Schmidt number, D is the diffusion of the solute, and dimensionless Schmidt number on dialysate side can be included in factor a. From these equations, the permeability coefficient of the solute through the membrane $\left(\mathrm{P}_{\mathrm{M}}\right)$ can be determined.

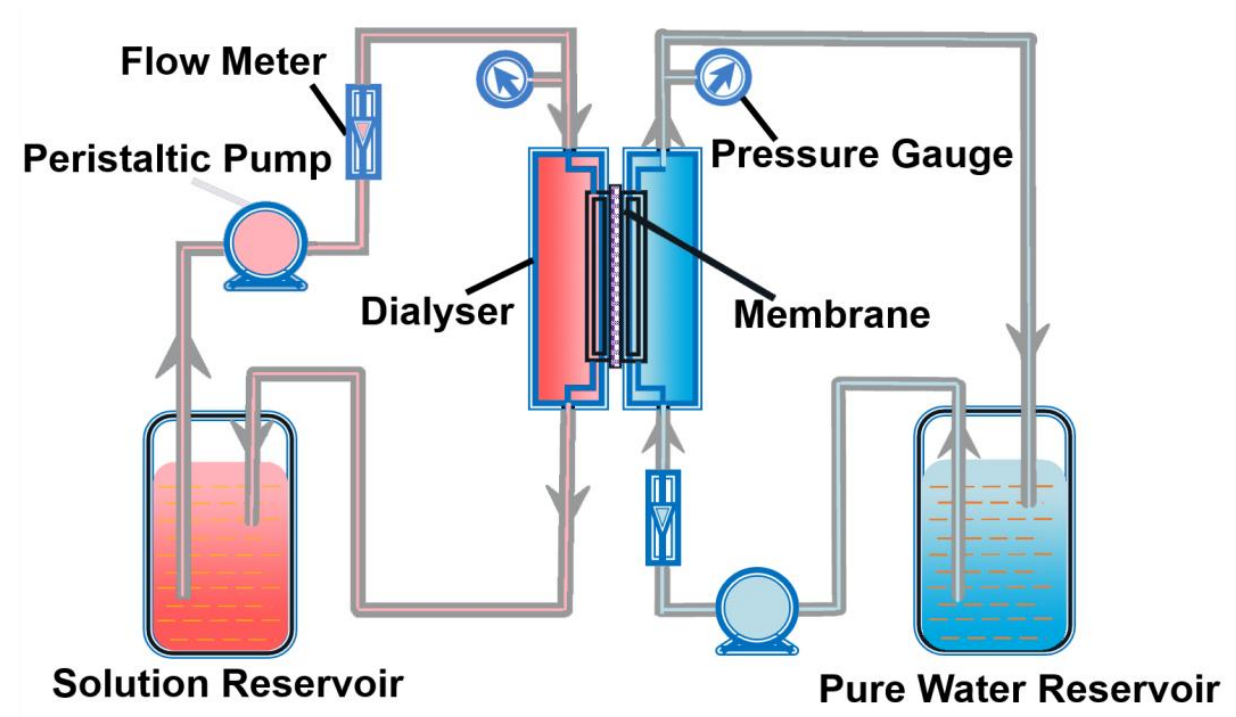

Fig. 1 Schematic diagram of lab-scale single membrane dialysis system.

\section{Results and discussion}

The characteristics of an ideal hemodialysis membrane (Fig. 2), as based on theoretical considerations of the transport through the membrane structure, can be defined as follows: (1) the porosity of support layer should be high and the active separation layer should be as thin as possible to achieve a high permeability; (2) the active separation layer should have a uniform pore-size distribution in order to 
achieve the greatest selectivity, and the maximum pore size should not exceed a certain limit to prevent loss of necessary proteins, for example, albumin; (3) hydrophilic surface properties for spontaneous wetting are needed to ensure low protein adsorption and superior blood compatibility. In addition to these basic requirements, the mechanical property of the membrane should be sufficient to withstand the pressures that occur during the treatment processes. Hence, we fabricated a novel nanocomposite hemodialysis membrane, based on the transport mechanism, which consisted of an ultrathin hydrophilic active separation layer and a scaffold-like nanofiber support layer, and the results were discussed in the following sections.

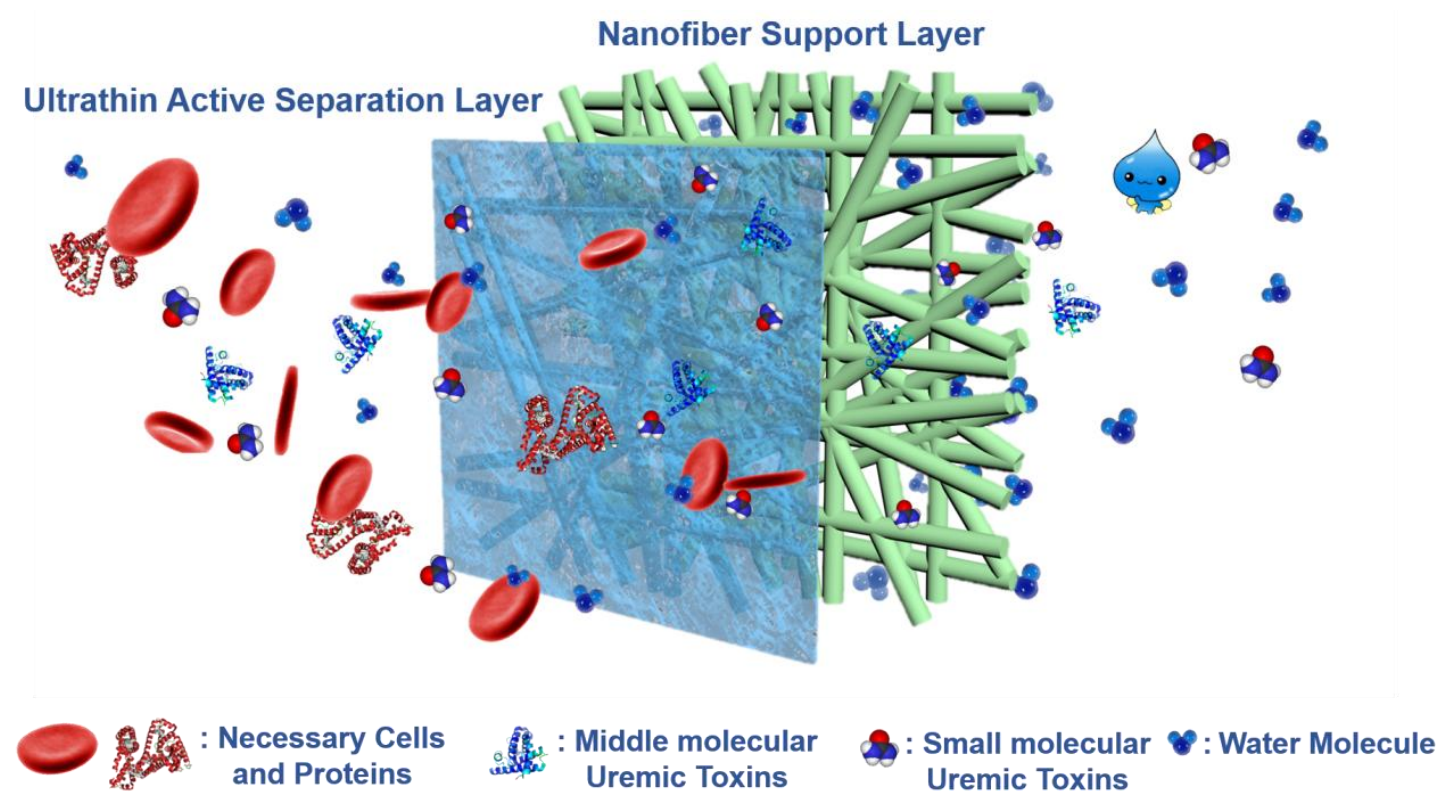

Fig. 2 Schematic of an ideal hemodialysis membrane (remove excess water, toxic metabolites and prevent loss of necessary proteins) with an active separation layer and a scaffold-like nanofiber support layer. The top layer is ultrathin and hydrophilic to achieve a high permeability and has a narrow pore-size distribution to ensure the greatest selectivity. The nanofiber support layer possesses highly interconnected pore structure, which provides direct paths for water and toxic metabolites excretion.

\subsection{Characterization of PAN nanofibrous scaffolds}


Electrospinning is a process based on electrohydrodynamics to produce a porous nanofiber matrix. This fabrication method is simple, easy, and cost-effective, and various polymers have been used in electrospinning processes. Among them, electrospun PAN membranes have attracted much attention due to their excellent thermal stability and insolubility to most solvents. Fig. 3A and B showed the typical SEM images of the PAN nanofibrous scaffold before and after cold-pressed. Scaffold prepared using $8 \%$ PAN solution showed the fiber-dominant morphology. The fiber diameter distribution was relatively uniform and the average diameter of the PAN fibers was about $255 \pm 30 \mathrm{~nm}$. The nanofibrous substrate was more compact after being pressed and the morphology of PAN nanofiber didn't change distinctly. It was found that the pores in an electrospun substrate were fully interconnected to form a three-dimensional network. Thus, the PAN nanofibrous scaffold was the excellent candidate as the supporting layer. The thickness of the prepared PAN nanofibrous supporting layer after cold-pressed was about $40 \pm 5 \mu \mathrm{m}$, and these scaffolds were used in the following experiments.
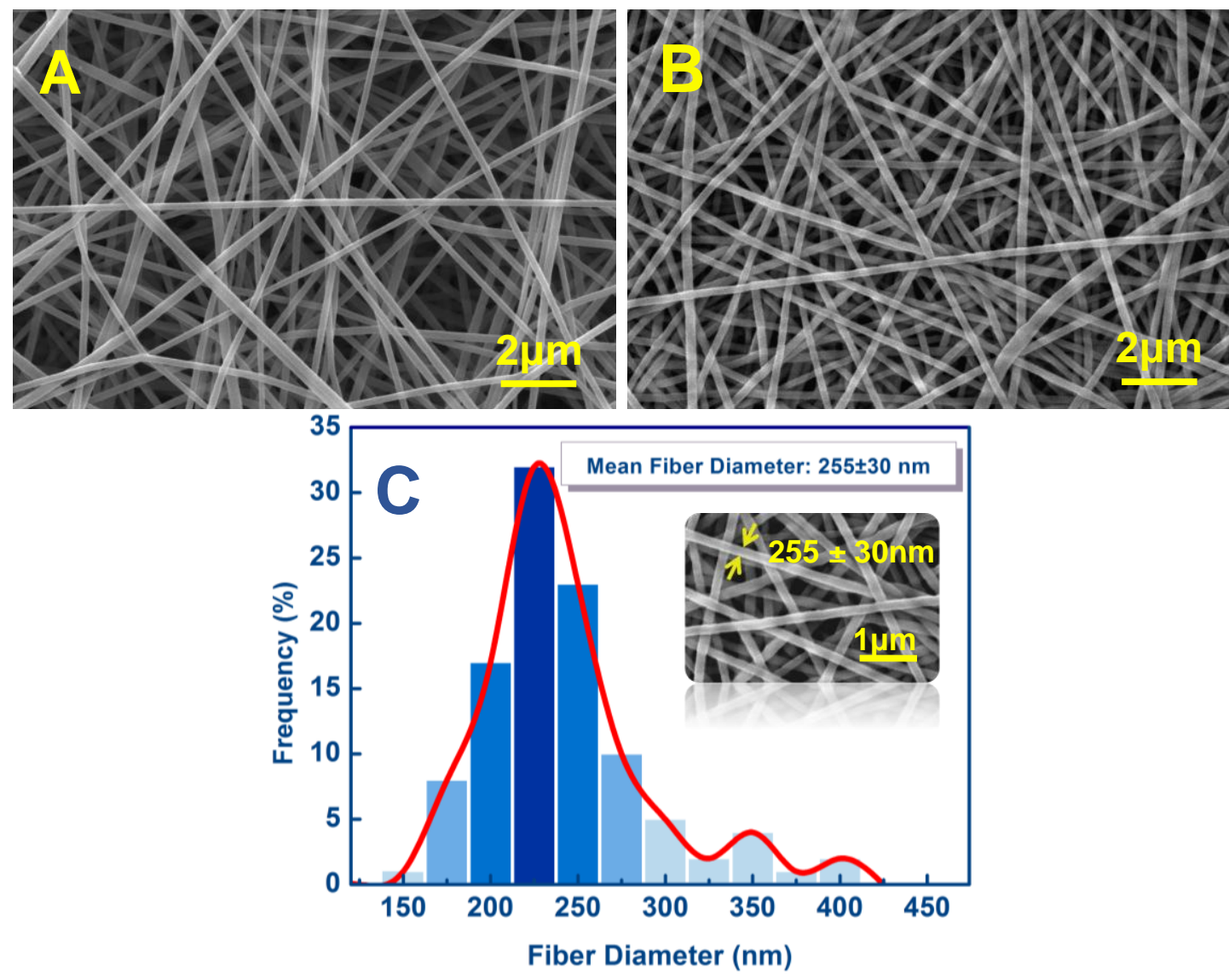

Fig. 3 Typical SEM images of PAN nanofibrous substrate electrospun from 8 wt $\%$ 
PAN/DMF solution before (A) and after (B) cold-pressed at $5 \mathrm{MPa}$, and fiber diameter distribution (C) of electrospun PAN nanofibrous scaffold.

\subsection{Optimization of PVA Active Separation Layer on Electrospun PAN Scaffold}

By definition, dialysis is a process of separating solutes in a solution by diffusion across a semipermeable membrane (diffusive solute transport) down a concentration gradient. Differences in permeability are reflected in the diffusive properties of the membrane. The overall resistance of transport is mainly contributed by membrane structure, and the diffusion properties of a dialysis membrane are dependent on the thickness of the active separation layer. Thus, it was very crucial to achieve a desirable minimum thickness of the PVA active separation layer for the preparation of TFNC hemodialysis membranes. Fig. 4 showed the surface SEM images of PAN nanofibrous scaffolds covered by cross-linked PVA separation layer with different coating solutions. When the concentration of PVA solution was lower than $2 \mathrm{wt} \%$, the PVA top separation layer was not perfectly covered on the PAN nanofibrous layer (as shown in Fig. 4A and B) which could be attributed to the penetration of the dilute PVA solution. Some regions were not covered by PVA layer and some small pores could be clearly seen in the SEM image. Increasing the concentration of PVA solution should resolve this problem. The integrated and nonporous PVA top layer with the concentration of $2 \mathrm{wt} \%$ could be observed in Fig. 4C and it became thicker (the impression of the nanofibers faded out) with the increase of concentration (Fig. 4D). The optimization of PVA active separation layer made it possible to create ultrathin active separation layer with favorable diffusive transport properties. 

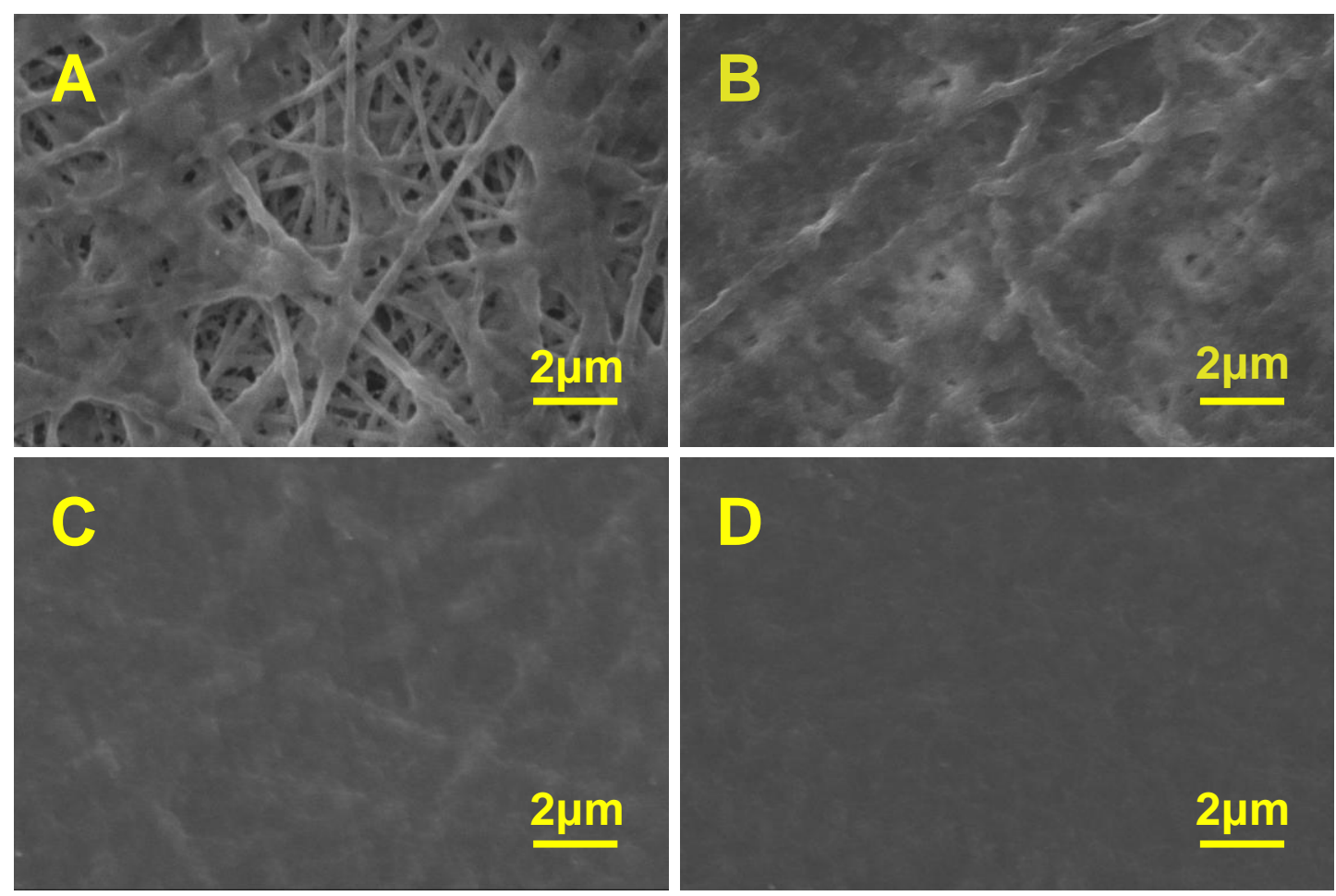

Fig. 4 The surface SEM images of TFNC membranes based on PVA coating and electrospun PAN scaffold prepared with different coating solutions ((A) 1 wt\%; (B) $1.5 \mathrm{wt} \%$; (C) $2 \mathrm{wt} \%$; and (D) $2.5 \mathrm{wt} \%$ ).

For hemodialysis purpose, a sufficient amount of permeation flux through the dialysis membrane is important for removing excess water from the patient's body. In addition, albumin is one of the useful substances, the leakage of which if left unchecked, may compromise the nutritional status of the patient. Thus, it is desirable to minimize the albumin loss during the dialysis process. In TFNC membranes, the top separation layer should be optimized for high permeability and great selective protein rejection capability. Cross-flow measurements were carried out to characterize the pure water permeation flux and protein rejection of the PVA/PAN TFNC membrane for membrane preparation regulating. The crosslinking reaction was triggered by an aldol condensation between aldehyde groups and hydroxyl groups at room temperature. When the PVA layer was crosslinked, the size of covalent bond bridged network was dependent on the degree of crosslinking. Thus, the increase in the degree of crosslinking in the separation layer would decrease the corresponding networks size, resulting in increasing the solute rejection ratio and decreasing the 
permeate flux. Fig. 5 showed the relations of pure water permeation flux and protein rejection of the composite membrane with the crosslinking degree of the PVA active separation layer. It could be seen that, high protein rejection $(95 \%)$ with high flux $\left(290 \mathrm{~L} / \mathrm{m}^{2} \mathrm{~h}\right.$ ) could be achieved when the GA/PVA repeat unit ratio was 0.25 . Further increase in the crosslinking degree of the PVA layer would continuously decrease the flux. Thus, the conditions of $2 \mathrm{wt} \%$ PVA solution and the GA/PVA repeat unit ratio of 0.25 were used in the following experiments and it was highly beneficial for them to be proposed as possible hemodialysis membranes.

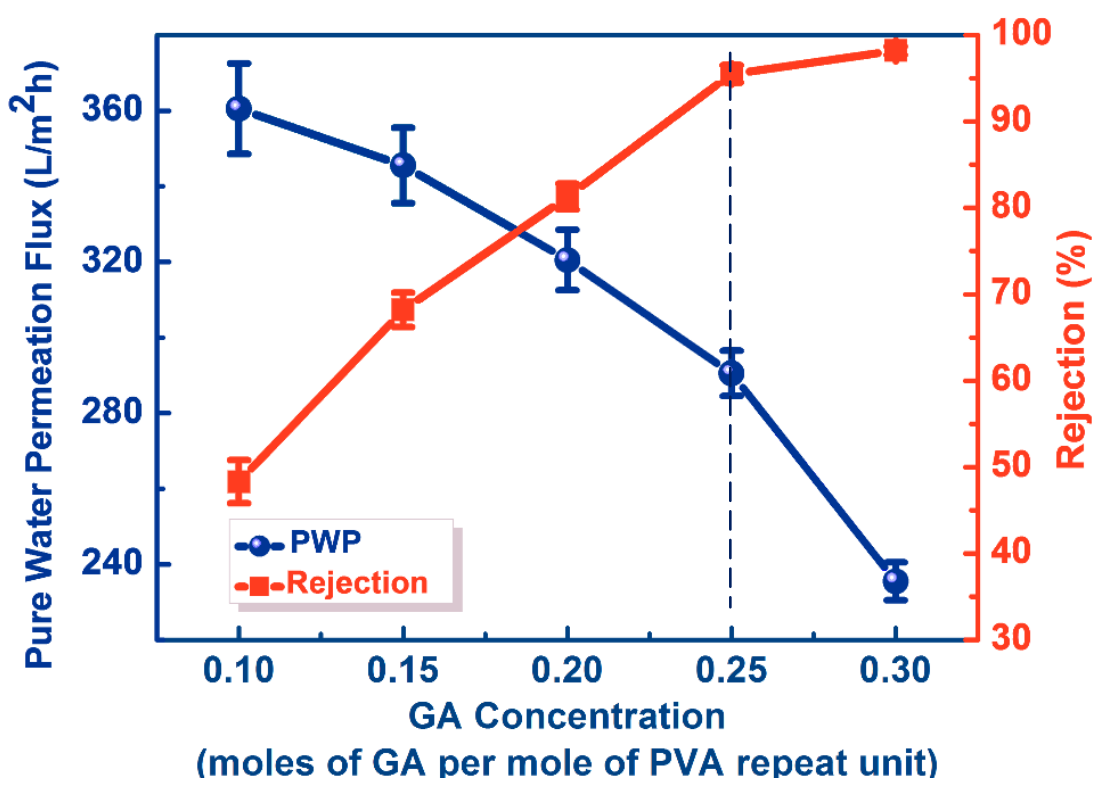

Fig. 5 Relations of pure water permeation flux and BSA rejection of the nanofibrous composite membranes with the degree of crosslinking of the PVA hydrogel coating (feed pressure: $0.1 \mathrm{MPa}$; temperature: $\left.30-35^{\circ} \mathrm{C}\right)$. Data were mean $\pm \mathrm{SD}(\mathrm{n}=3)$.

\subsection{Membrane morphologies}

The membrane performance is closed related to the morphology of the membrane. In-depth understanding of the membrane formation process and structure is necessary for the improvement of membrane performance, such as permeation rate and selectivity. For comparison, conventional benchmark flat sheet PSf membrane was prepared and adopted as the control sample. As shown in Fig. 6A, the cross-sectional SEM image of the conventional PSf membrane showed the typical three-tier structure 
formation of a phase inversion membrane, i.e. a separation skin layer, a finger-like structure support layer, and a sponge-like bottom layer. The mutual affinity between the solvent and non-solvent was high, leading to instantaneous demixing and finger-like structures formation. A slow exchange rate between solvent and non-solvent, resulted in sponge-like structures in membrane morphology. Similar membrane structures made from various polymers have been described before [17-19]. Conventional membranes fabricated by phase separation generally exhibited low pore densities, closed cell pore structure and relatively high thickness of active separation layer (a skin layer and a spongy bottom layer). The closed cell porous structure and thick spongy bottom layer might block the toxic middle molecular substances through the membrane and exhibited low clearance for middle molecules.

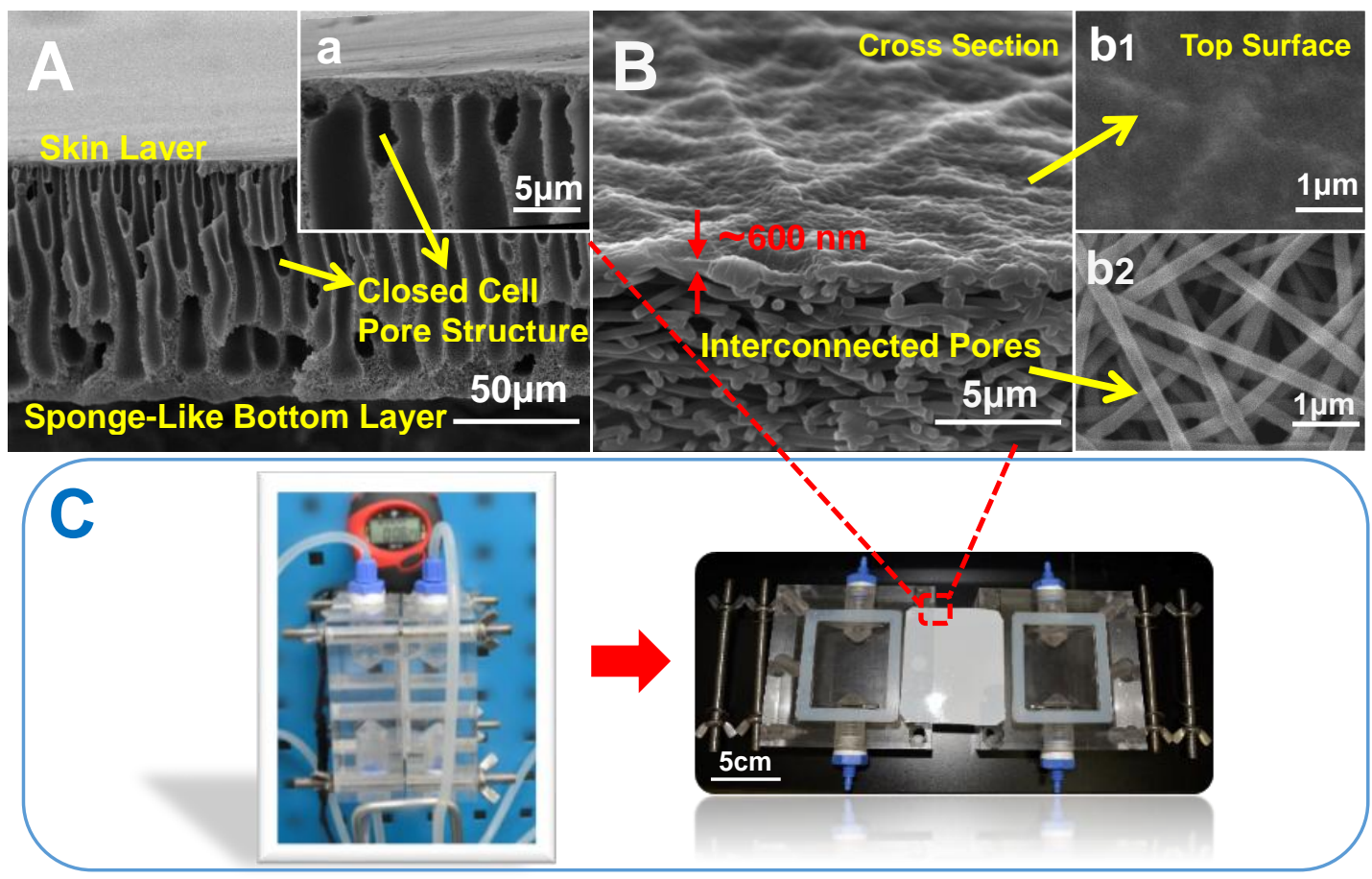

Fig. 6 (A) A cross-sectional SEM image of a conventional benchmark PSf membrane using the phase inversion method showed the tortuous sponge-like closed cell pore structure. (B) The typical cross-sectional SEM image of the PVA/PAN TFNC membrane. The top surface SEM showed that the electrospun substrate was covered with an ultrathin coating layer. The cross-sectional SEM image showed that the scaffold had low-tortuosity structure and the pores were interconnected between individual nanofibers. (C) Optical photographs of PVA/PAN TFNC membrane and 
dialysis membrane cell assembly.

In contrast, Fig. 6B showed the cross-sectional SEM image of the PVA/PAN TFNC membrane. The nanofiber support layer showed porous structure with interconnected pores between individual nanofibers, so there were many paths along which the particles could move on. This was very different from the conventional membranes where many dead-end pores existed from the phase inversion process. The unique structure of the nanofiber support layer guaranteed direct paths for water and toxic metabolites excretion, which could provide a good solution for eliminating the permeability bottleneck. The PVA integrated active separation layer with thickness of about $600 \mathrm{~nm}$ was very thin. The top surface SEM image (Fig. $6 b_{1}$ ) showed the typical ridge-and-valley morphology of the PVA active layer on electrospun PAN substrate membranes. The TFNC membrane had high porosity, interconnected porous supporting layer, and ultrathin coating layer, so superior removal efficiencies and selectivity can be expected.

\subsection{Sieving curve}

Recent studies focused on the removal of "middle-molecule toxins", including important uremic toxins. $\beta_{2}$-microglobin, a protein of 11,600 Daltons, accumulates in hemodialysis patients resulting in amyloid formation and the amyloid deposition, mainly presents as destructive arthropathy $[47,48]$. Because of the severity and the destructive nature of the complication, it is very important to efficiently remove the $\beta_{2}$-microglobin from the plasma. On the other hand, high albumin retention is one of the important requirements of a hemodialysis membrane in order to avoid hypoalbuminemia on patients during hemodialysis. Thus, the membrane should possess appropriate sieving properties. In other words, hemodialysis membranes should facilitate the permeability of uremic toxins of small and middle molecular weights and simultaneously reject the transport of proteins. Fig. 7 presented the sieving curves of conventional benchmark PSf and PVA/PAN TFNC membranes. It 
was important to note that the PVA/PAN TFNC membrane had a quite sharper separation curve than that of the conventional PSf membrane. For the molecules urea (60 Da) and lysozyme (14 kDa), the SC value was approximately 1.0 and 0.75 , respectively, while the $\mathrm{SC}$ value for $\mathrm{BSA}(66 \mathrm{kDa})$ was around 0.05 . It could be speculated that this might be due to a uniform pore-size distribution of PVA/PAN TFNC membrane. The above results indicated that the PVA/PAN TFNC membrane provided great selectivity for hemodialysis.

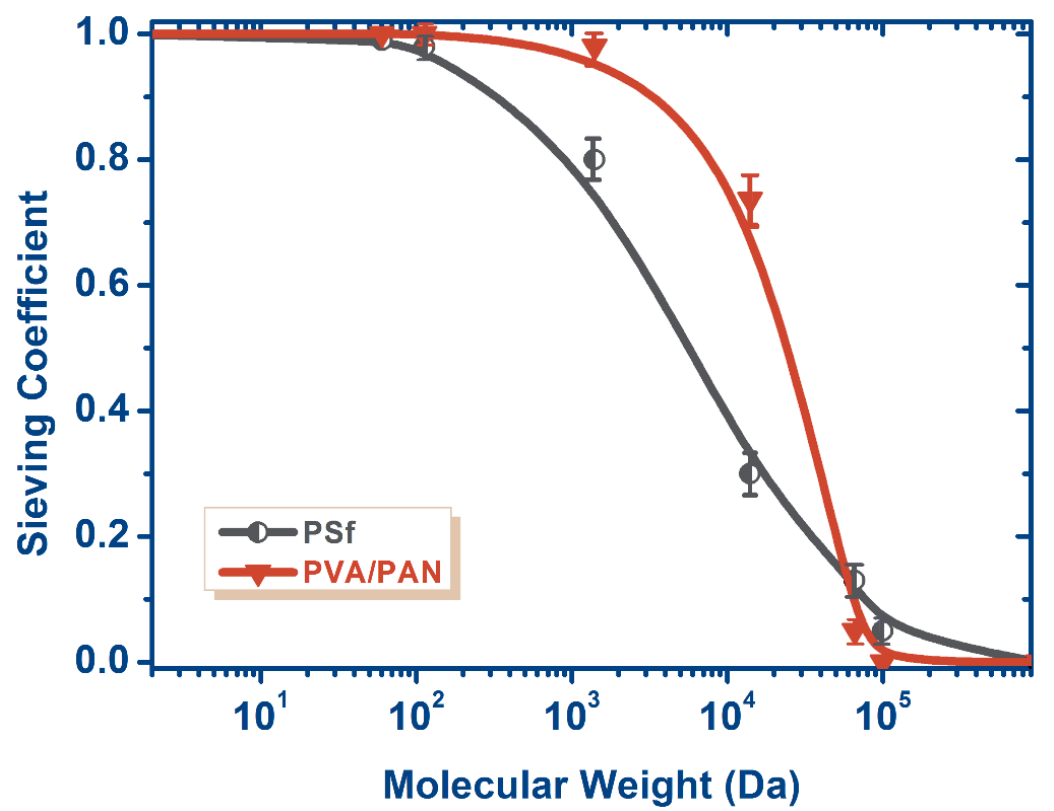

Fig. 7 Sieving coefficients of molecular weight markers for the conventional benchmark PSf and PVA/PAN TFNC membranes. The following markers were used: urea (60 Da), creatinine (112 Da), vitamin $\mathrm{B}_{12}(1355 \mathrm{Da})$, lysozyme $(14 \mathrm{kDa})$ and $\mathrm{BSA}(66 \mathrm{kDa})$. Data were mean $\pm \mathrm{SD}$.

\subsection{Hydrophilicity}

The water contact angles of different membranes were measured to characterize the hydrophilic characteristics. The contact angle for hydrophilic membranes often attenuates with the elongation of drop age because of the pores and chemical structures of the membranes. The dynamic water contact angle variations of the conventional benchmark PSf and PVA/PAN TFNC membranes were measured and 
the results were shown in Fig. 8. Typically, the smoother membrane surface was considered to be advantageous. However, it could be seen that although the smoother PSf membrane surface compared to the TFNC membrane (the surface showed ridge-and-valley morphology), the PSf membrane initial contact angle was $78.9^{\circ}$ due to its highly hydrophobic, and the initial contact angle of the PVA/PAN TFNC membrane was lower $\left(67.3^{\circ}\right)$ than that of PSf membrane due to the hydrophilic hydroxyl group on PVA. Furthermore, the curve of water contact angle for the PVA/PAN TFNC membrane attenuated much faster with drop age than that of the conventional PSf membrane. The contact angle of the PVA/PAN TFNC membrane rapidly attenuated to $55^{\circ}$ within $60 \mathrm{~s}$. As contrast, the contact angle of the PSf membrane changed only about $2^{\circ}$ within drop age of $120 \mathrm{~s}$. The fast decreasing contact angle for the PVA/PAN TFNC membrane was mainly attributed to the high surficial hydrophilicity. Water droplets could easily penetrate the surface of hydrophilic membranes. The hydrophilic surface of the PVA/PAN TFNC membrane was in favor of the permeability and could decrease the protein adsorption and suppress platelet adhesion, thus improve the blood compatibility, which will be discussed in detail in the followings.
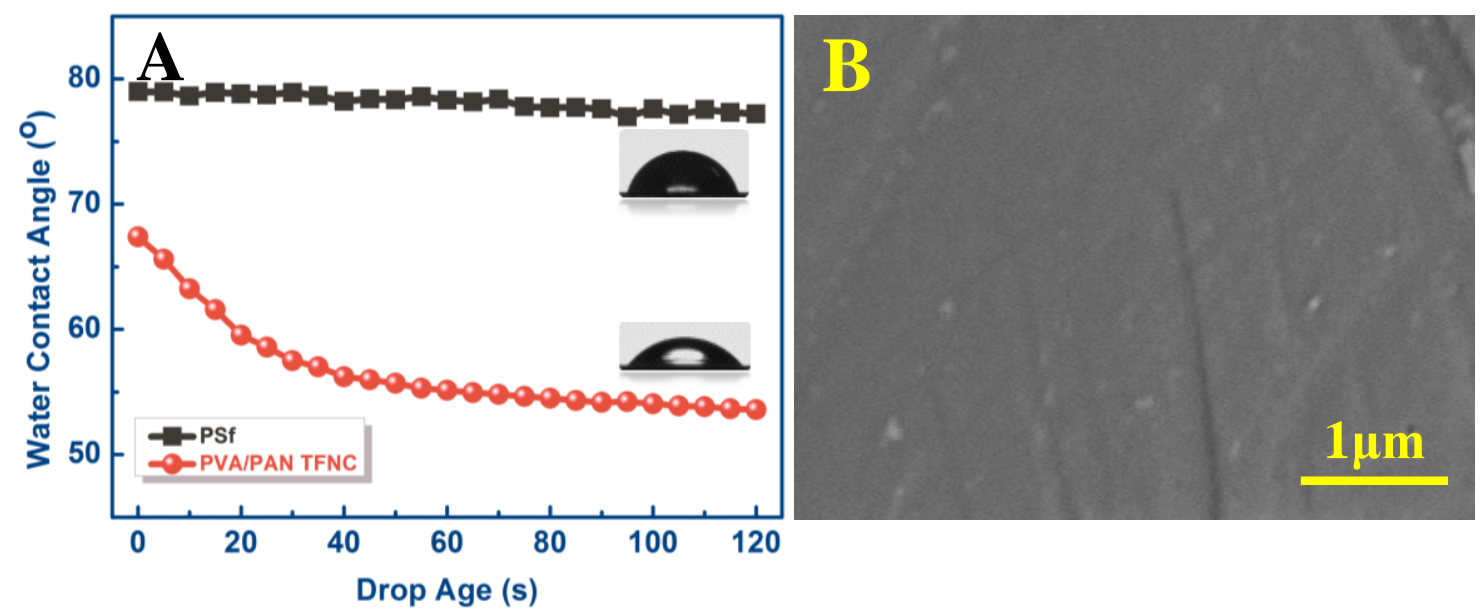

Fig. 8 (A) Dynamic water contact angle decaying with drop age for the conventional benchmark PSf, PVA/PAN TFNC membranes and the representative pictures of water contact angles. (B) The surface SEM images of PSf membrane. 
The mechanical properties of the conventional benchmark PSf and the PVA/PAN TFNC membranes have been investigated by tensile tests. Fig. 9 showed the tensile stress and strain curves of the conventional PSf membrane (curve A), the electrospun PAN nanofibrous supporting layer before and after cold-pressed (curves B and C), and PVA/PAN TFNC membrane (curve D), respectively. The conventional PSf membrane we prepared in laboratory had a tensile stress of $5.4 \mathrm{MPa}$ and tensile strain of $28 \%$. This was consistent with the work of Gao et al [28]. It was obvious that the mechanical properties of electrospun PAN supporting layer were improved significantly by cold-press, and the PVA/PAN TFNC membrane showed good overall mechanical strength (the tensile strength of about 13.9 MPa and elongation at break of $55 \%)$. It could be concluded that the electrospun nanofibrous substrate not only provided good permeability but also the mechanical strength for the TFNC membranes. As a result, the PVA/PAN TFNC membrane had high mechanical performance for hemodialysis membranes.

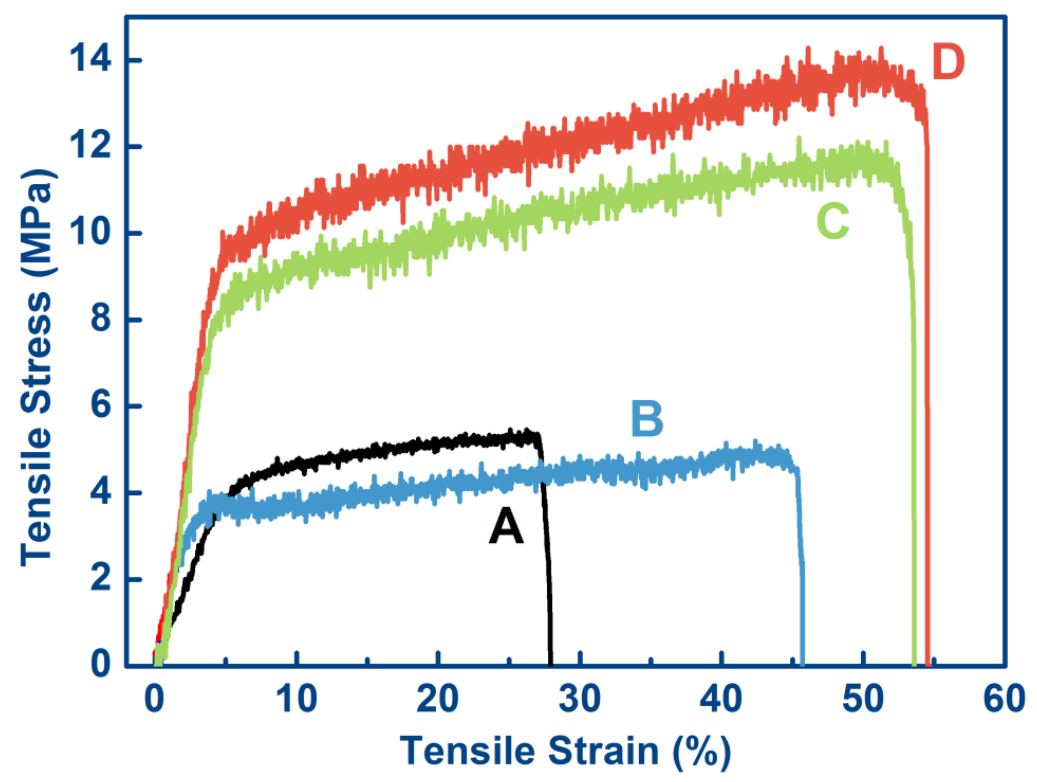

Fig. 9 Tensile stress-strain curves of the conventional benchmark PSf membrane (A), electrospun PAN nanofibrous supporting layer before (B) and after cold-pressed (C), and PVA/PAN TFNC membrane (D). 


\subsubsection{Protein adsorption}

Any blood-contacting material initially adsorbs the plasma proteins and may give rise to thrombus formation. Therefore, hemocompatibility is a significant aspect for blood-contacting materials. The adsorption of proteins is one of the key issues in evaluating the hemocompatibility of the membranes. Here, protein adsorption was measured under static condition. As we know, hydrophobic membrane surface adsorbs more proteins irreversibly from plasma when it comes into contact with blood, which can induce permeability decrease and lead to bigger complications, such as complement activation. Hydrophilicity helps to form a thin layer of aqueous film on the surface of the membrane, impeding the deposition of proteins on the membrane surface and leading to less adsorption of protein. Therefore, the more hydrophilic membranes are supposed to have less protein adsorption. The protein adsorption behavior of the sample membranes incubated with BSA solution was displayed in Fig. 10. The hydrophilic PVA/PAN TFNC membrane showed lower protein adsorption $\left(28.9 \pm 0.3 \mu \mathrm{g} / \mathrm{cm}^{2}\right)$ as compared to the control PSf membrane $\left(42.8 \pm 0.5 \mu \mathrm{g} / \mathrm{cm}^{2}\right)$.

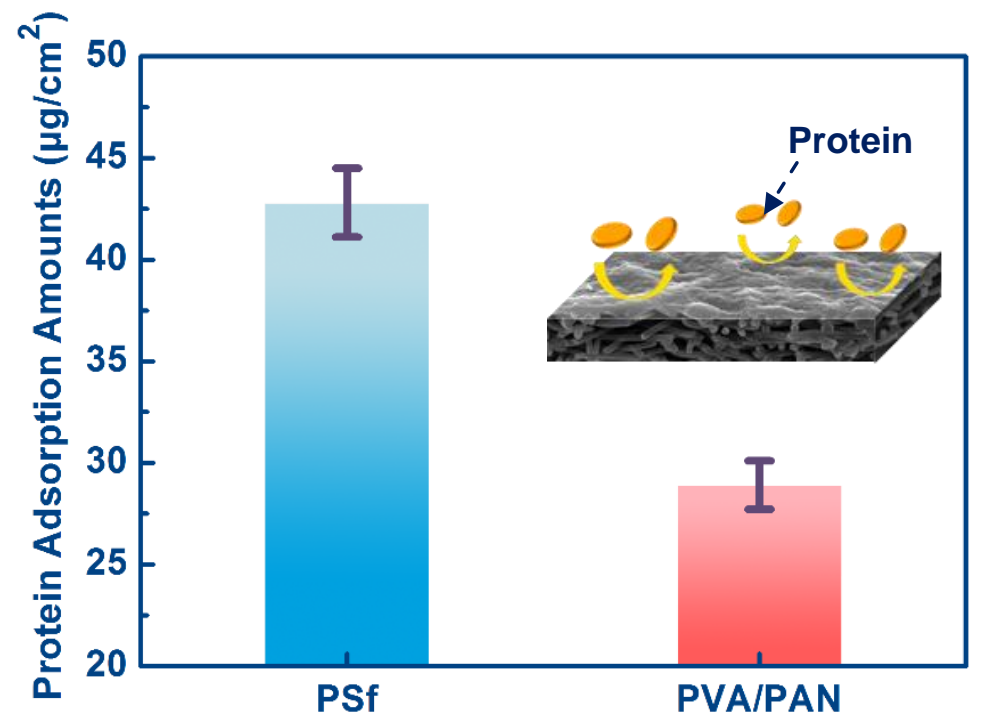

Fig. 10 BSA adsorption amounts onto the membranes. Values were expressed as means $\pm \mathrm{SD}, \mathrm{n}=5$.

\subsubsection{Platelet adhesion}


Platelet adhesion on the membrane surface from plasma and the morphology of the adhering platelets are considered as key events in thrombus formation. Thus, another important factor to evaluate blood compatibility is platelet adhesion. It was observed that the platelets adhering on the conventional benchmark PSf membrane were aggregated in large numbers on the membrane surface and possessed a network-like structure with deformed and extended pseudopodium, which indicated that potential thrombus formation might be induced at the material interface (Fig. 11A). A small number of platelets were observed on the TFNC membrane and the adhered platelets displayed sphere-like shapes with almost no pseudopodia or deformation (Fig. 11B).The results were consistent with the previous protein adsorption results. The reduction of the platelet adhesion could be attributed to the introduction of the hydrophilic surface layer.

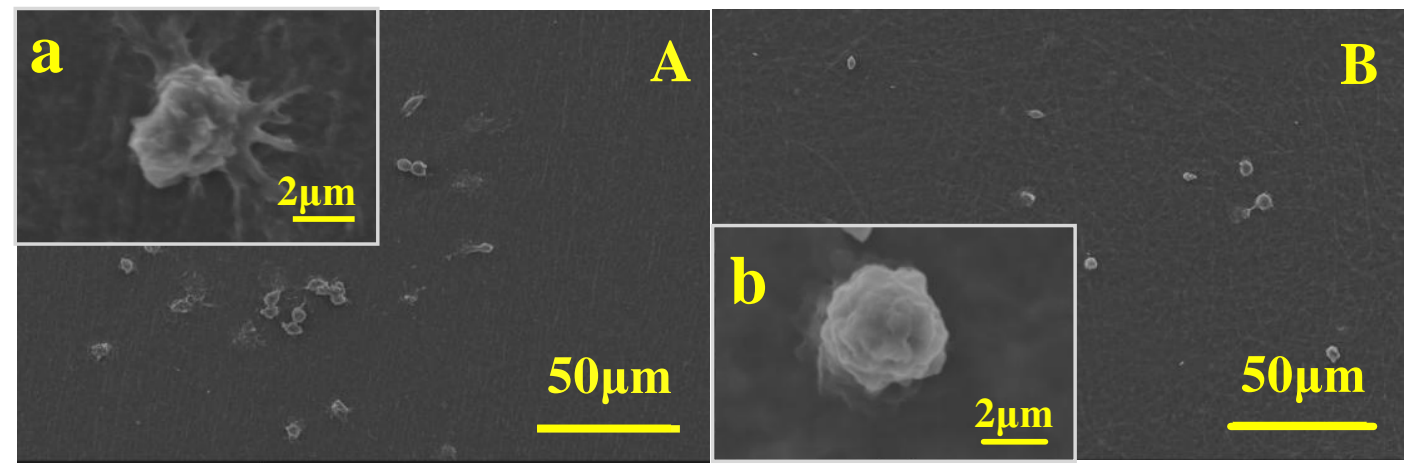

Fig. 11. SEM micrographs of the platelets adhering on the conventional benchmark PSf membrane (A) and TFNC membrane (B).

\subsubsection{Complement activation}

Complement activation is the body's defense mechanism against pathogens. Thus, it is another vital aspect of hemocompatibility. After complement activation, the anaphylatoxins $\mathrm{C} 3 \mathrm{a}$ and $\mathrm{C} 5 \mathrm{a}$ are liberated and then combined with specific cell surface receptors and triggers the host defense mechanism by generating the localized inflammatory mediator. Thus, it could be quantified by the measurement of the produced anaphylatoxins $\mathrm{C} 3 \mathrm{a}$ and $\mathrm{C} 5 \mathrm{a}$. As shown in Fig. 12A, both the conventional benchmark PSf membrane and the TFNC membrane showed no significant difference 
from the control sample in the C3a levels $(<0.5 \%, * \mathrm{p}>0.05)$. For the C5a levels (Fig. 12B), the concentration of C5a for the conventional benchmark PSf membrane was slightly higher than that of the control sample, whereas the TFNC membrane showed no significant difference from the control sample. Fig. 12 presented the results of complement activation, the TFNC membrane showed comparable properties to the conventional benchmark PSf membrane.
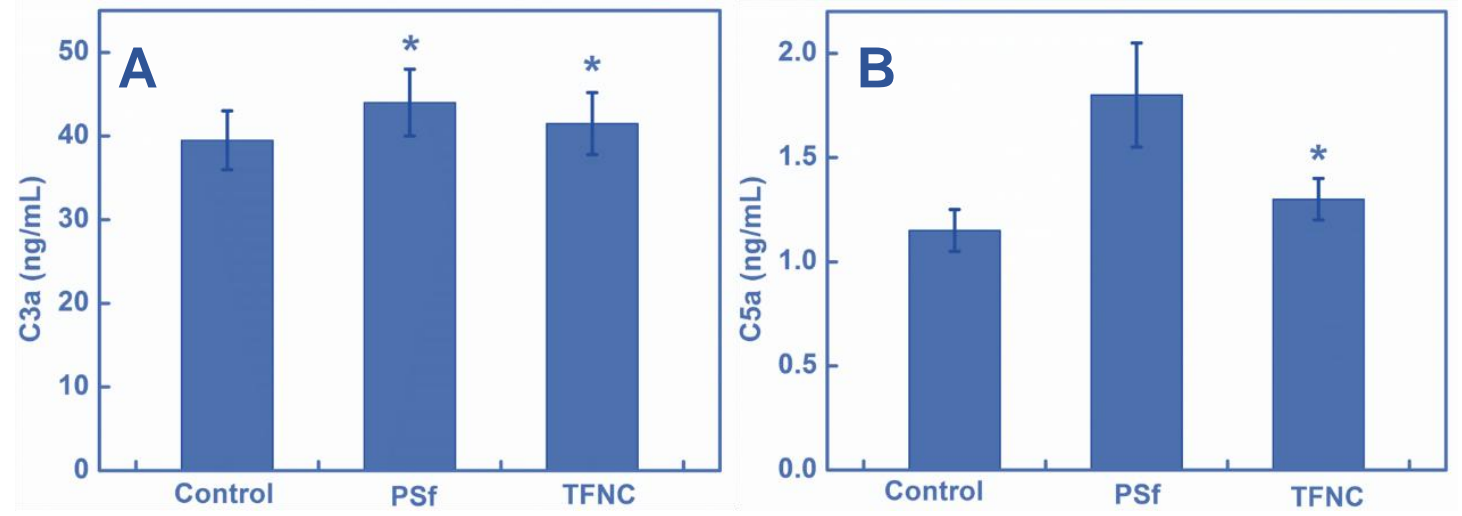

Fig. 12. (A) Concentration of $\mathrm{C} 3 \mathrm{a}$ for the samples with whole blood. (Values are expressed as mean \pm SD.) (B) Concentration of C5a for the samples with whole blood. (Values are expressed as mean $\pm \mathrm{SD}$. $* \mathrm{p}>0.05$ compared with plasma.)

\subsubsection{Hemolysis}

Hemolysis assay is often used as a screening test to assess hemocompatibility and it is generally used to detect the erythrocyte damage caused by materials. Hemolysis less than 5\% is regarded as non-toxic according to the standard. Table 1 presented the average hemolysis ratio of the sample membranes. As could be seen from that, the conventional benchmark PSf membrane had the considerable hemolysis ratio of $3.18 \%$. Correspondingly, the PVA/PAN TFNC membrane did not induce toxic levels of hemolysis in this in vitro hemolysis-screening test and showed better results $(1.36 \%)$ than the conventional PSf membranes. This result indicated that the PVA/PAN TFNC membrane was safe as blood contact materials. 
Table 1 Hemolysis ratio of different sample membranes.

\begin{tabular}{lll}
\hline Sample & Absorbance (540nm) & Hemolytic Rate (\%) \\
\hline Positive Reference & $5.3144 \pm 0.0150$ & - \\
Negative Reference & $0.1305 \pm 0.0010$ & - \\
Benchmark PSf & $0.2954 \pm 0.0015$ & 3.18 \\
PVA/PAN TFNC & $0.2012 \pm 0.0015$ & 1.36 \\
\hline
\end{tabular}

\subsection{Hemodialysis}

PSf is often used by the industry for the preparation of commercial hemodialysis membranes. Therefore we selected benchmark PSf membranes (home-made) for comparison with our two-tier TFNC membrane. The urea and lysozyme clearance, BSA retention of the conventional PSf and PVA/PAN TFNC membranes were measured to evaluate the hemodialysis performances. From Fig. 13A, it could be figured out that $82.6 \%$ of urea was cleaned by the PVA/PAN TFNC membrane after simulating dialysis for $4 \mathrm{~h}$, which was higher than $76.2 \%$ of the conventional PSf membrane. With respect to middle molecule toxic clearance, $45.8 \%$ of lysozyme was cleaned out for the PVA/PAN TFNC membrane, which was about 3.5 times higher than that of the conventional PSf membrane (12.8\%) after simulating dialysis for $4 \mathrm{~h}$ (Fig. 13B). As described in the introduction session, the albumin lose should be avoided for the dialysis membrane design so as to prevent albumin lost associated syndrome such as hypoalbuminemia during hemodialysis process. The dialysis TFNC membrane produced in this study was very reliable, and valuable blood protein was scarcely lost (98.8\% retention) during the dialysis process, while the BSA retention for the conventional PSf membrane was about 91.2\% (Fig. 13C). 

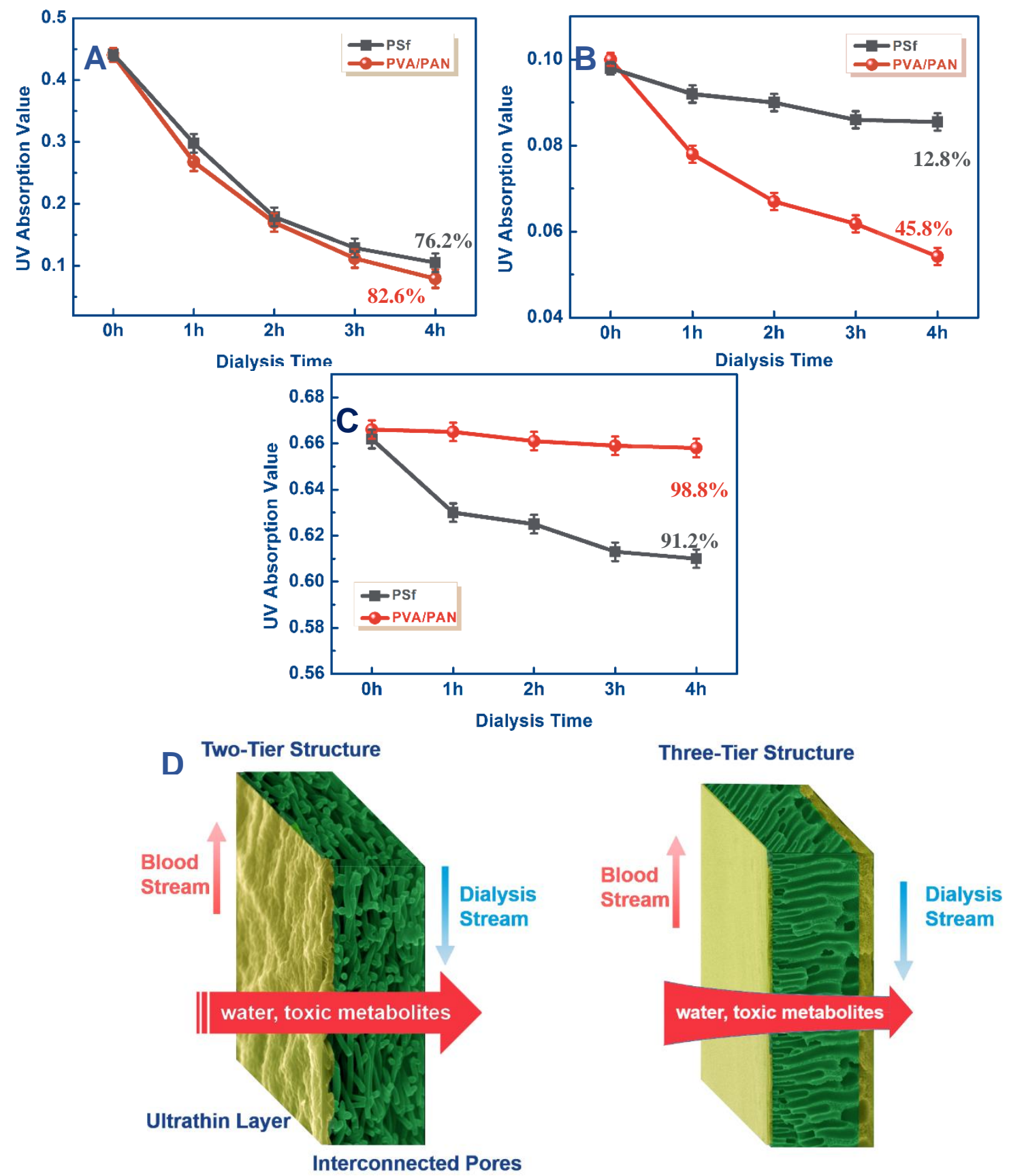

Fig. 13 Comparison of the solute transmission efficiency for the conventional benchmark PSf and PVA/PAN TFNC membranes: (A) urea clearance percentage; (B) lysozyme clearance percentage; (C) BSA retention percentage. The error bars indicated standard deviations. (D) Comparison of the permeability for conventional membrane and TFNC membrane. The ultrathin coating layer and open pore structure of scaffold favored diffusion and convection. In the conventional membrane, the tortuous, closed structure dramatically decreased permeability. 
Table 2 illustrated a detailed comparison of dialysis performances among the present work and recently reported literatures. From these data it could be seen that these neat polymer membranes were much difficult for the removal of middle-molecule toxin (1.2\% for pure PES membrane after simulating dialysis for $4 \mathrm{~h}$ and $12.6 \%$ for pure PLA membrane). In order to improve the removal efficiency of middle molecules toxin, many attempts have been made to increase pore size and improve the permeability via surface grafting, coating or blending (17.9\% for heparin immobilized PLA membrane, $27.9 \%$ for PES/PVP/MWCNT membrane and $33.0 \%$ for PEO-PLA membrane). Furthermore, these membranes showed significant loss of albumin ( $90 \%$ retention). As a result, conventional hemodialysis membrane is still inadequate to remove middle-molecules while retaining albumin. According to the previous equation, overall resistance to mass transfer consists of blood, membrane and dialysate components. It is mainly contributed to the membrane resistance. Membrane resistance could be directly inferred from the membrane structure (Fig. 13D). In reality, current hemodialysis membranes fabricated via one step phase inversion method not only presented a skin layer for separating big proteins and small/middle toxics, but also possessed another "skin" layer (thick sponge-like bottom layer) in the opposite of the membrane. This sponge-like structure of bottom layer was formed with small pores segregated by dense walls, which would also be the main obstacle for toxics diffusion. In addition, the tortuous closed cell pore structure in the support layers might also decrease the permeability despite the fact that finger-like pores could span most of conventional membrane thickness. It was paradoxical to reconcile the desire of high removal of middle molecular toxic substances and high retention of big proteins at the same time for the conventional hemodialysis membrane. However, the PVA active separation layer of two-tier TFNC membranes could achieve a desirable minimum thickness of hydrophilic layer, and the open pore structure of nanofiber support layer favored the diffusion and convection. Hence, the uremic toxins, which were dissolved in water, were carried with water and passed through the membrane more easily. This seemed to be supported by the results for urea and lysozyme. In addition, the permeability 
coefficients of the solutes are in concordance with their molecular weight. It is clear that middle molecules toxin passes freely through the TFNC membrane while big albumin is almost completely blocked and these results were much more effective than conventional hemodialysis membranes reported so far. It can be explained using the classical membrane transport theory and the comparison of experimental with models validated a correlation of permeability through the membrane. And it was indicated that the two-tier TFNC membrane had a potential wide application as the next generation of hemodialysis membrane.

Table 2 Comparison of membrane properties and dialysis performances with recently reported literatures.

\begin{tabular}{|c|c|c|c|c|c|c|}
\hline \multirow[b]{2}{*}{ Membranes } & \multirow[b]{2}{*}{ Year } & \multirow{2}{*}{$\begin{array}{l}\text { Pure water } \\
\text { permeation } \\
\text { flux } \\
(\mathrm{L} / \mathrm{m} 2 \mathrm{~h})\end{array}$} & \multicolumn{3}{|c|}{ Dialysis performances } & \multirow[b]{2}{*}{$\begin{array}{l}\text { Ref. } \\
\text { no. }\end{array}$} \\
\hline & & & $\begin{array}{l}\text { Urea } \\
\text { clearance } \\
(\%)\end{array}$ & $\begin{array}{l}\text { Lysozyme } \\
\text { clearance } \\
(\%)\end{array}$ & $\begin{array}{l}\mathrm{BSA} \\
\text { retention } \\
(\%)\end{array}$ & \\
\hline Chitosan-g-PVAc & 2012 & l & 37.5 & I & $\sim 98$ & [20] \\
\hline Pure PVDF & 2014 & 5.10 & 10.0 & l & $\sim 98$ & {$[15]$} \\
\hline PVDF/PEG & 2014 & 27.5 & 62.0 & l & $\sim 94$ & {$[15]$} \\
\hline Pure PES & 2014 & 7.10 & 11.5 & 1.2 & $\sim 90$ & {$[18]$} \\
\hline Pure PLA & 2014 & 120.0 & 74.6 & 12.6 & 93.7 & [19] \\
\hline $\begin{array}{l}\text { Heparin } \\
\text { immobilized PLA }\end{array}$ & 2014 & 109.5 & 79.0 & 17.9 & 90.8 & [19] \\
\hline PES/PVP/MWCNT & 2014 & 68.5 & 56.3 & 27.9 & $\sim 90$ & [18] \\
\hline PLA/PEO & 2015 & 224.5 & 77.0 & 33.0 & 90.9 & [28] \\
\hline $\begin{array}{l}\text { Benchmark PSf } \\
\text { (home-made) }\end{array}$ & 2016 & 105 & 76.2 & 12.8 & 91.2 & l \\
\hline TFNC (this study) & 2016 & 290.5 & 82.6 & 45.8 & 98.8 & l \\
\hline
\end{tabular}




\section{Conclusions}

In summary, a new type of high-performance hemodialysis membrane consisting of an ultrathin hydrophilic active separation layer and a scaffold-like nanofiber support layer was demonstrated. This represented the first attempt of PVA/PAN two-tier TFNC membranes for their potential application in hemodialysis. Naturally, the ultrathin hydrophilic PVA skin layer provided high selectivity (a narrow pore-size distribution) to remove uremic toxins and retain proteins, the nanofibrous support layer offered high permeability (a highly interconnected pore structure) to transfer the toxins during hemodialysis process. Two-tier PVA/PAN exhibited $98.8 \%$ blood protein retention, $82.6 \%$ urea clearance, and $45.8 \%$ lysozyme clearance respectively. TFNC membranes exhibited excellent dialysis performances, especially for the middle-molecule uremic toxin removal, which were much more effective than conventional hemodialysis membranes reported so far.

It is imperative to point out that the above two-tier TFNC membranes have not been optimized, and the chosen material system (i.e. PVA for active separation layer) may not be the most appropriate one for the artificial kidney in practice (e.g. compared with commercial available hemodialysis membranes due to their hollow fiber configuration). Although we do not have data to address this concern, we believe that the demonstrated two-tier structured TFNC membrane paves a new way to fabricate high performance hemodialysis media which could well reconcile the desire of high removal of middle-molecular toxic substances and high retention of big proteins simultaneously. For the future development of TFNC membranes, other hemodialysis materials and their modification will be optimized to achieve better blood compatibility and higher efficient dialysis performance. In addition, careful design of the dialysis module must be needed to use the TFNC membranes in a commercial-module, the related design and evaluation of multi-flat tandem dialysis module will be the subjects of our future study.

\section{Acknowledgments}


This work was supported by National Science Foundation of China (51273042, 21174028), Program for New Century Excellent Talents in University (NCET-13-0725), Program of Shanghai Science and Technology Innnovation International Exchange and Cooperation (15230724700), Program of Changjiang Scholars and Innovative Research Team in University (IRT1221).

\section{References}

[1] S.S. Hayek, S. Sever, Y.A. Ko, H. Trachtman, M. Awad, S. Wadhwani, M.M. Altintas, C. Wei, A.L. Hotton, A.L. French, L.S. Sperling, S. Lerakis, A.A. Quyyumi, J. Reiser, Soluble urokinase receptor and chronic kidney disease, N. Engl. J. Med. 373 (2015) 1916-1925.

[2] C.C. Striemer, T.R. Gaborski, J.L. McGrath, P.M. Fauchet, Charge- and size-based separation of macromolecules using ultrathin silicon membranes, Nature 445 (2007) 749-753.

[3] C. Legallais, G. Catapano, B.V. Harten, U. Baurmeister, A theoretical model to predict the in vitro performance of hemodiafilters, J. Membr. Sci. 168 (2000) 3-15.

[4] A. Zelman, D. Gisser, G. Strait, V. Bastidas, R. Stephen, C. Kablitz, J. Harrow, B. Deeter, W.J. Kolff, Pressure control of the ultrafiltration rate during hemodialysis with high-flux dialyzers and the time dependence of membrane transport parameters, ACS Symp. Ser. 154 (1981) 61-74.

[5] P.J. Blankestijn, I. Ledebo, B. Canaud, Hemodiafiltration: clinical evidence and remaining questions, Kidney Int. 77 (2010) 581-587.

[6] H. Humes, W. Fissell, K. Tiranathanagul, The future of hemodialysis membranes, Kidney Int. 69 (2006) 1115-1119.

[7] S. Xia, N. Hodge, M. Laski, T.F. Wiesner, Middle-molecule uremic toxin removal via hemodialysis augmented with an immunosorbent packed bed, Ind. Eng. Chem. Res. 49 (2010) 1359-1369.

[8] S. Acchiardo, A.P. Kraus, B.R. Jennings, $\beta_{2}$-Microglobulin levels in patients with 
renal insufficiency, Am. J. Kidney Dis. 13 (1989) 70-74.

[9] B.H. Su, P. Fu, Q. Li, Y. Tao, Z. Li, H.S. Zao, C.S. Zhao, Evaluation of polyethersulfone highflux hemodialysis membrane in vitro and in vivo, J. Mater. Sci.: Mater. Med. 19 (2008) 745-751.

[10] A. Idris, K.L. Yet, The effect of different molecular weight PEG additives on cellulose acetate asymmetric dialysis membrane performance, J. Membr. Sci. 280 (2006) 920-927.

[11] C.M. Kee, A. Idris, Permeability performance of different molecular weight cellulose acetate hemodialysis membrane, Sep. Purif. Technol. 75 (2010) 102-113.

[12] A. Roy, P. Dadhich, S. Dhara, S. De, In vitro cytocompatibility and blood compatibility of polysulfone blend, surface-modified polysulfone and polyacrylonitrile membranes for hemodialysis, RSC Adv. 5 (2015) 7023-7034.

[13] F. Ran, S.Q. Nie, W.F. Zhao, J. Li, B.H. Su, S.D. Sun, C.S. Zhao, Biocompatibility of modified polyethersulfone membranes by blending an amphiphilic triblock co-Polymer of poly(vinyl pyrrolidone)-b-poly(methyl methacrylate)-b-poly(vinyl pyrrolidone), Acta Biomater. 7 (2011) 3370-3381.

[14] T. Xiang, L.S. Zhang, R. Wang, Y. Xia, B.H. Su, C.S. Zhao, Blood compatibility comparison for polysulfone membranes modified by grafting block and random zwitterionic copolymers via surface-initiated ATRP, J. Colloid Interface Sci. 432 (2014) 47-56.

[15] K.H. Chan, E.T. Wong, M.I. Khan, A. Idris, N.M. Yusof, Fabrication of polyvinylidene difluoride nano-hybrid dialysis membranes using functionalized multiwall carbon nanotube for polyethylene glycol (hydrophilic additive) retention, J. Ind. Eng. Chem. 20 (2014) 3744-3753.

[16] Y. Chang, W.J. Chang, Y.J. Shih, T.C. Wei, G.H. Hsiue, Zwitterionic sulfobetaine-grafted poly(vinylidene fluoride) membrane with highly effective blood compatibility via atmospheric plasma-induced surface copolymerization, ACS Appl. Mater. Interfaces 3 (2011) 1228-1237.

[17] L.J. Zhu, F. Liu, X.M. Yu, L.X. Xue, Poly(lactic acid) hemodialysis membranes with poly(lactic acid)-block-poly(2-hydroxyethyl methacrylate) copolymer as additive: 
preparation, characterization, and performance, ACS Appl. Mater. Interfaces 7 (2015) 17748-17755.

[18] M. Irfan, A. Idris, N.M. Yusof, N.F.M. Khairuddin, H. Akhmal, Surface modification and performance enhancement of nano-hybrid f-MWCNT/PVP90/PES hemodialysis membranes, J. Membr. Sci. 467 (2014) 73-84.

[19] A.L. Gao, F. Liu, L.X. Xue, Preparation and evaluation of heparin-immobilized poly(lactic acid) (PLA) membrane for hemodialysis, J. Membr. Sci. 452 (2014) 390-399.

[20] C. Radhakumary, P.D. Nair, C.P.R. Nair, S. Mathew, Chitosan-graft-poly(vinyl acetate) for hemodialysis applications, J. Appl. Polym. Sci. 125 (2012) 2022-2033.

[21] L. Li, L. Schulte, L.D. Clausen, K.M. Hansen, G.E. Jonsson, S. Ndoni, Gyroid nanoporous membranes with tunable permeability, ACS Nano 10 (2011) 7754-5566.

[22] M. Radjabian, V. Abetz, Tailored pore sizes in integral asymmetric membranes formed by blends of block copolymers, Adv. Mater. 27 (2015) 352-365.

[23] D. Rana, T. Matsuura, Surface modifications for antifouling membranes, Chem. Rev. 110 (2010) 2448-2471.

[24] M. Tijink, J. Janssen, M. Timmer, J. Austen, Y. Aldenhoff, J. Kooman, L. Koole, J. Damoiseaux, R.V. Oerle, Y. Henskens, D. Stamatialis, Development of novel membranes for blood purification therapies based on copolymers of n-vinylpyrrolidone and n-butylmethacrylate, J. Mater. Chem. B 1 (2013) 6066-6077. [25] N.J. Kaleekkal, D. Rana, D. Mohan, Functionalized MWCNTs in improving the performance and biocompatibility of potential hemodialysis membranes, RSC Adv. 6 (2016) 63156-63170.

[26] N.J. Kaleekkal, A. Thanigaivelan, M. Durga, R. Girish, D. Rana, P. Soundararajan, D. Mohan, Graphene oxide nanocomposite incorporated poly(ether imide) mixed matrix membranes for in vitro evaluation of its efficacy in blood purification applications, Ind. Eng. Chem. Res. 54 (2015) 7899-7913.

[27] L.J. Zhu, F, Liu, X.M. Yu, A.L. Gao, L.X. Xue, Surface zwitterionization of hemocompatible poly(lactic acid) membranes for hemodiafiltration, J. Membr. Sci. 475 (2015) 469-479. 
[28] A.L. Gao, F. Liu, H.Y. Shi, L.X. Xue, Controllable transition from finger-like pores to inter-connected pores of PLLA membranes, J. Membr. Sci. 478 (2015) 96-104.

[29] X.X. Song, Z.Y. Liu, D.D.Sun, Nano gives the answer: breaking the bottleneck of internal concentration polarization with a nanofiber composite forward osmosis membrane for a high water production rate, Adv. Mater. 23 (2011) 3256-3260.

[30] H.Y. Ma, B.S. Hsiao, B. Chu, Functionalized electrospun nanofibrous microfiltration membranes for removal of bacteria and viruses, J. Membr. Sci. 452 (2014) 446-452.

[31] X.F. Wang, X.M. Chen, K. Yoon, D.F. Fang, B.S. Hsiao, B. Chu, High flux filtration medium based on nanofibrous substrate with hydrophilic nanocomposite coating, Environ. Sci. Technol. 39 (2005) 7684-7691.

[32] X.F. Wang, D.F, Fang, K. Yoon, B.S. Hsiao, B. Chu, High performance ultrafiltration composite membranes based on poly(vinyl alcohol) hydrogel coating on crosslinked nanofibrous poly(vinyl alcohol) scaffold, J. Membr. Sci. 278 (2006) 261-268.

[33] K. Yoon, B.S. Hsiao, B. Chu, High flux ultrafiltration nanofibrous membranes based on polyacrylonitrile electrospun scaffolds and crosslinked polyvinyl alcohol coating, J. Membr. Sci. 338 (2009) 145-152.

[34] H. You, Y. Yang, X. Li, K. Zhang, X.F. Wang, M.F. Zhu, B.S. Hsiao, Low pressure high flux thin film nanofibrous composite membranes prepared by electrospraying technique combined with solution treatment, J. Membr. Sci. 394 (2012) 241-247.

[35] L.D. Shen, X.F. Yu, C. Cheng, C.L. Song, X.F. Wang, M.F. Zhu, B.S. Hsiao, High filtration performance thin film nanofibrous composite membrane prepared by electrospraying technique and hot-pressing treatment, J. Membr. Sci. 499 (2016) 470-479.

[36] W.S. Dai, T.A. Barbari, Gel-impregnated pore membranes with mesh-size asymmetry for biohybrid artificial organs, Biomaterials 21 (2000) 1363-1371.

[37] S. Sakai, M. Tsumura, M. Inoue, Y. Koga, K. Fukano, M. Taya, Polyvinyl 
alcohol-based hydrogel dressing gellable on-wound via a co-enzymatic reaction triggered by glucose in the wound exudate, J. Mater. Chem. B 1 (2013) 5067-5075.

[38] M. Irfan, A. Idris, Overview of PES biocompatible/hemodialysis membranes: PES-blood interactions and modification techniques, Mater. Sci. Eng., C 56 (2015) 574-592.

[39] S. Senthilkumar, S. Rajesh, A. Jayalakshmi, G. Aishwarya, D.R. Mohan, Preparation and performance evaluation of poly(ether-imide) incorporated polysulfone hemodialysis membranes, J. Polym. Res. 19 (2012) 9867-9878.

[40] F.Y. Mahlicli, S.A. Altinkaya, Surface modification of polysulfone based hemodialysis membranes with layer by layer self-assembly of polyethyleneimine/alginate-heparin: a simple polyelectrolyte blend approach for heparin immobilization, J. Mater. Sci.: Mater. Med. 24 (2013) 533-546.

[41] H.Y. Ma, K. Yoon, L.X. Rong, M. Shokralla, A. Kopot, X. Wang, D.F. Fang, B.S. Hsiao, B. Chu, Thin-film nanofibrous composite ultrafiltration membranes based on polyvinyl alcohol barrier layer containing directional water channels, Ind. Eng. Chem. Res. 49 (2010) 11978-11984.

[42] J. Deng, X.Y. Liu, L. Ma, C. Cheng, W.B. Shi, C.X. Nie, C.S. Zhao, Heparin-mimicking multilayer coating on polymeric membrane via LbL assembly of cyclodextrin-based supramolecules, ACS Appl. Mater. Interfaces 6 (2014) 21603-21614.

[43] W. Zou, H. Qin, W.B. Shi, S.D. Sun, C.S. Zhao, Surface Modification of Poly(ether sulfone) Membrane with a Synthesized Negatively Charged Copolymer, Langmuir 30 (2014) 13622-13630.

[44] H.L. Wei, L.L. Han, J. Ren, L.Y. Jia, Anticoagulant surface coating using composite polysaccharides with embedded heparin-releasing mesoporous silica, ACS Appl. Mater. Interfaces 5 (2013) 12571-12578.

[45] L.J. Langsdorf, A.L. Zydney, Diffusive and convective solute transport through hemodialysis membranes: A hydrodynamic analysis, J. Biomed. Mater. Res. 28 (1994) 573-582.

[46] M. Raff, M. Welsch, H. Göhl, H. Hildwein, M. Storr, B. Wittner, Advanced 
modeling of highflux hemodialysis, J. Membr. Sci. 216 (2003) 1-11.

[47] R.K. Spencer, A.G. Kreutzer, P.J. Salveson, H. Li, J.S. Nowick, X-ray crystallographic structures of oligomers of peptides derived from $\beta_{2}$-microglobulin, $\mathrm{J}$. Am. Chem. Soc. 137 (2015) 6304-6311.

[48] C. Menaa, E. Esser, S.M. Sprague, $\beta_{2}$-Microglobulin stimulates osteoclast formation, Kidney Int. 73 (2008) 1275-1281. 


\section{Graphical Abstract}
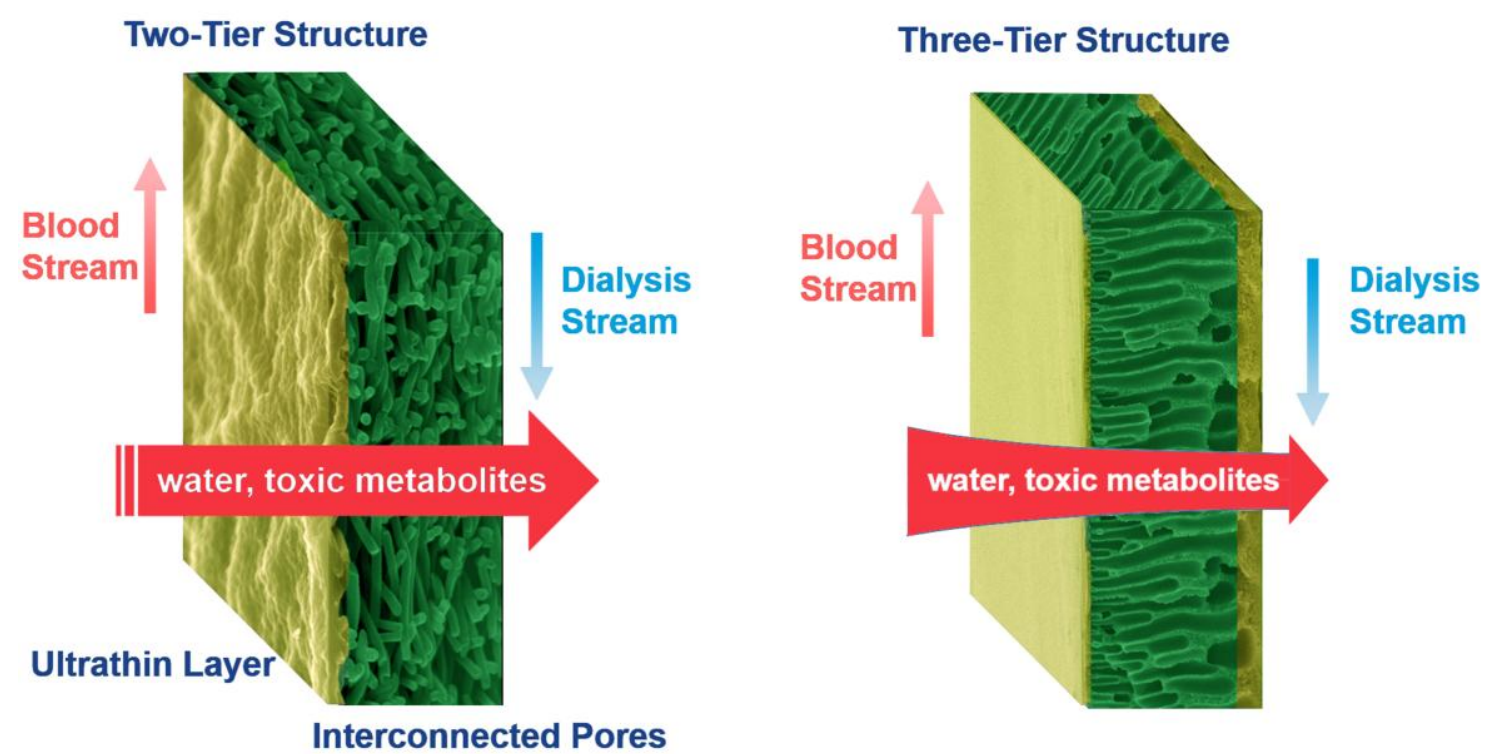